\title{
Numerical simulation and experiments on turbulent air flow around the semi- circular profile at zero angle of attack and moderate Reynolds number
}

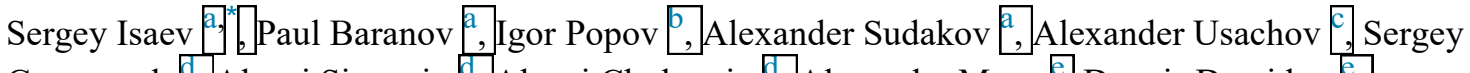
Guvernyuk ", Alexei Sinyavin ", Alexei Chulyunin ", Alexander Mazo ?, Dennis Demidov है, Alexander Dekterev ${ }^{\mathrm{tI}, \mathrm{g}}$, Andrey Gavrilov ${ }^{\mathrm{t}, \mathrm{g}}$, ,Alexander Shebelev $\mathrm{ft}$

${ }^{\mathrm{a}}$ Saint-Petersburg State University of Civil Aviation, Saint-Petersburg 196210, Russia

${ }^{\mathrm{b}}$ A. N. Tupolev Kazan National Research Technical University (Kazan Aviation Institute), Kazan 420111, Russia ${ }^{\mathrm{c}}$ N. E. Zhukovskii Central Aerohydrodynamic Institute, Moscow 107005, Russia

$\mathrm{d}$ Lomonosov Moscow State University Institute of Mechanics, Moscow 119192, Russia

e Kazan Federal University, Kazan 420008, Russia

${ }^{\mathrm{f}}$ Siberian Federal University, Krasnoyarsk 660041, Russia

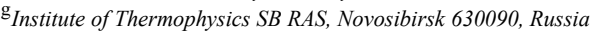

\section{abstract}

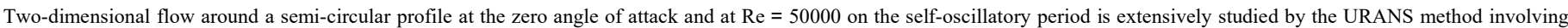

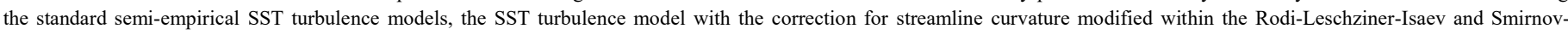

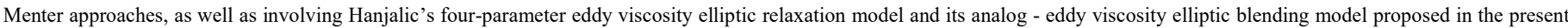

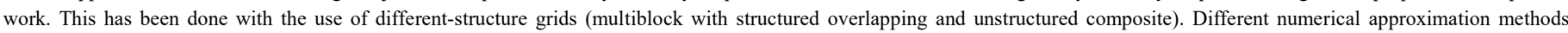

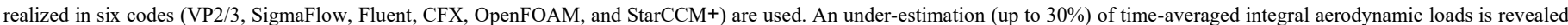

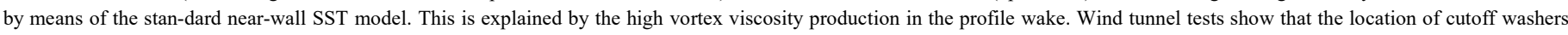

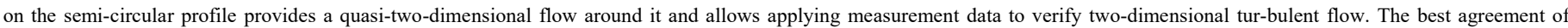

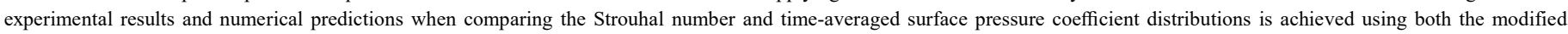

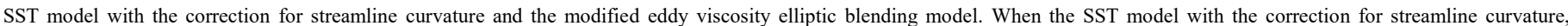

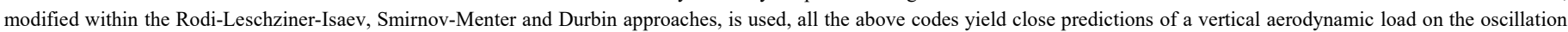
period.

\section{Introduction}

Interest in turbulent flow around a semi-circular profile is as-sociated with the features of its aerodynamic characteristics at the zero angle of attack $\alpha=0$. At this position of the profile, the time-averaged lift coefficient $C_{y}$ takes peak negative values of the order of -1 , and small deviations of $\alpha$ in the direction of increasing or decreasing its values sharply increase $C y$ up to positive values
The reason for such a behavior is explained by transforming the $C_{y}$ fluctuation period-averaged pattern of vortex flow around the pro-file [3]. On the other hand, the semi-circular profile can be consid-ered as a thick ( $50 \%$ of the chord) profile. In this respect, interest in this profile is to find perspective aerodynamic shapes of integrated vehicles with flow control by vortex cells [4-6]. The present work applies the data on flow with periodic vortex structures near a cir-cular cylinder at $\mathrm{Re}=50000$ [7-9] and on turbulent flow around a thin (12\% of the chord) NACA0012 airfoil at small, moderate, and large angles of attack [10]. 


\section{Nomenclature}

a blending function

$A_{i}, C_{i}, c_{i}, C_{\mu}, c_{\mu}$ constants of turbulence models

$C_{y}$

$C_{c}$

$C_{p}$

Err

$f_{c}$

$f$

F

fr

$k$

L

$L_{t}$

l

$p$

$P$

PSD

$R$

$R_{x}$

$R_{y}$

$\operatorname{Re}$

$\mathrm{Ri}_{t}$

$S$

$S_{i j}$

Sh

$t$

$\mathrm{Tu}$

$T_{t}$

$U$

$u, v$

$\overline{u_{i}^{\prime} u_{j}^{\prime}}$

$v^{2}$

$x, y$

$X$

$y^{+}$

$\alpha$

$\delta_{\mathrm{ij}}$

$\varepsilon$

$\varepsilon_{22}, \phi_{22}$

$\zeta$

$\mu$

$\rho$

$\omega$

$\Omega$

Subscripts

max, min maximum and minimum values

$t \quad$ turbulent values

* time kept from the start of the self-oscillatory cycle

$\infty \quad$ incoming flow parameters
The current work presents the results of testing and comparative analysis of the computational technology for solution of unsteady Reynolds-averaged Navier-Stokes (URANS) equations applied for simulation of vortex and separated flows in multiply connected and many-scale computational domains by the example of lowvelocity air flow around a semi-circular profile at the zero angle of attack and $\mathrm{Re}=50000$.

The choice of this example as a test task is due to the abovementioned anomalous $C_{y}$ behavior of such a profile at $\alpha=0$, since numerical simulation errors of intense vortex flow in this case appear to be most noticeable. These errors mainly depend on the chosen computational grids and the used semi-empirical turbulence models. Computational accuracy and efficiency are also affected by discretization methods, solution methods of algebraic equations, and their implementation in software - computational codes. All the above factors are reflected in the present testing study. Numerical predictions are verified by their comparison to the results of a wind tunnel special experiment.

As known, grids meant for solving boundary-value problems of fluid dynamics are divided into structured grids with ordered tetragonal (two-dimensional case) cells and unstructured grids with triangular, hexagonal cells, etc. [11]. For viscous fluid flows with high gradients (with boundary and shear layers) at high Reynolds numbers (Re), the characteristic zone scales are inversely proportional to Re. To correctly resolve them, mesh nodes should be condensed and computational cell sizes should be substantially decreased. To resolve vortex streets in the body wake, cells should also be refined. Fully unstructured grids are poorly adapted to condense mesh nodes in the vicinity of walls; the computational accuracy of these grids according to high-order schemes is lower than that according to structured grids. At the same time, it should be noted that some interesting tasks of aeromechanics are characterized by the presence of multiply connected computational subdomains, for example, flow around the profile with vortex cells [4,5], with trailing-edge flaps, and leading-edge flaps [12,13], flow interactions with a cylinder in a perforated housing when air from a high-pressure zone flows into a low-pressure zone in the wake [14]. In the case of complex flow boundary configurations, the use of single-block (with one matrix) structured grids runs into difficulties; therefore, multiblock overlapping grids have been long used. Dependent variables on such grids should be interpolated in near-boundary cells. This, as a rule, leads to the violation of mass and momentum conservation. This fact, as well as the presence of coarse cells at the periphery of fragment-composed grids led to the fact that in 1996, "DRAGON"-type grids [12], where overlapping zones of grids were discarded and overlapping grids were replaced by unstructured ones, were proposed for tasks of flow around a composite profile.

Despite the obvious promise of the concept, the above "DRAGON" -type grids were developed and used in applied software. At the same time, the development of the multiblock computational technology (MCT) in the VP2/3 code (velocity-pressure, 2D/3D) united the application of overlapping grids for multiply connected computational domains and the introduction of additional difference-scale grids for boundary and shear layers, vortex streets, and other physical phenomena of tasks at hand [15]. So in [16], where the task on circulation flow in a cavity with a movable cover was solved on single- and multiblock overlapping grids, the solution error was assessed using the linear interpolation in near-boundary cells of fragment-composed grids and its applicability was shown. In the present work, the predictions with the use of different-type grids are combined with the implication of different methods and applied software.

Semi-empirical constants in the turbulence models used for URANS closure as a rule are calibrated according to the measurement data for near-wall flows. The success of most models is de- 
termined through their use for low-intense turbulent vortex and separated flows tasks. However, it has long been known [17] that it is necessary to correct the two-parameter dissipative model with the consideration of the streamline curvature influence on turbulence characteristics when calculating flows with intense largescale trapped vortices. In recent decades, Menter's shear stress transport turbulence model (SST model) has been widely used for simulation of separated flows. It is included into all known commercial and open (open access code) applied software. Of the two SST models proposed by Menter in 1993 [18] and by Menter et al. in 2003 [19] software uses the last one. To predict eddy viscosity, the first model applies the absolute vorticity and the second the strain rate tensor modulus. Distinction between these models is significant and is seen in predictions of large-scale vortices. It is known that in vortex cores, vorticity is almost constant (Batchelor's model [20]]. Therefore, when the first model is used, vortex viscosity does not increase in the cores. At the same time, as confirmed by numerous tests [21,22], the use of the SST model [19] yields a false eddy viscosity increase in the core of largescale vortices and a very high eddy viscosity level as a whole. To avoid a non-physical eddy viscosity increase, this became possible within the generalized Rodi-Leschziner-Isaev (RLI) approach [15] with the correction for eddy viscosity computed by the SST model [19] with the correction function of the turbulent Richardson number when the value of Isaev-Kharchenko-Usachov's semiempirical constant $C_{c}=0.02$ is taken instead of 0.1 [17] in the correction for the two-parameter dissipative model. The correction for streamline curvature within the RLI approach is introduced into VP2/3 and SigmaFlow codes. The current work also tests the SST model [19] modified by Smirnov and Menter [23] on the basis of the ideas of Spalart and Shur [24]. This SST model [19] with the correction for streamline curvature within the Smirnov-Menter (SM) approach is used in Fluent, CFX, and VP2/3 codes. And, at last, when the StarCCM+code is applied, Durbin's correction [25] is added to the SST model.

The standard SST model [19] is verified by means VP2/3, SigmaFlow, Fluent, CFX, OpenFOAM, and StarCCM+ codes used for computation of turbulent flow around a semi-circular profile at $\alpha=0^{\circ}$ and $\operatorname{Re}=50000$. The versions of the SST model with the streamline curvature correction, the four-parameter $\zeta-f$ model [26], and the four-parameter $\zeta-a$ model, developed on the basis of works [26-29], were also tested in combination with the use of different-topology grids and various computational meth- ods when solving this task. The numerical predictions by means of these computational models are compared with the results of a wind tunnel special experiment to define local and integral aerodynamic loads on the semi-circular profile.

\section{Physical model}

As mentioned above, a semi-circular cylinder can be considered as a thick (50\% of the chord) airfoil. In our case, flow around such an airfoil at zero angle of attack is taken as a basic example to test computational technologies of two-dimensional numerical simulation of turbulent flows. At that, it is important that in experiment, appropriate flow around a semi-circular cylinder should also be quasi-two-dimensional.

Work [1] was concerned with the experimental study of flow around the semi-circular cylinder at different angles of attack $\alpha$, including at $\alpha=0$, and at $\operatorname{Re} \geq 67000$. Time-averaged distributions of the pressure coefficient $C_{p}$ over the contour of the cross section of the semi-circular cylinder were obtained and integral aerodynamic forces were assessed. However work [1] has no data, showing whether flow around the semi-circular cylinder was quasi-twodimensional or whether it was substantially three-dimensional. In the present study, new experimental data on flow around the semi-circular cylinder at zero angle of attack were obtained using special means to provide quasi-two-dimensional flow.

Fig. 1 shows the experimental design for measurement of pressure distributions over the cross section of the semi-circular cylinder in the uniform flow at zero angle of attack. Measurements were made in the wind tunnel with the cross section shaped as a regular octagon with a side of $320 \mathrm{~mm}$. The diameter of the semicircular cylinder was $L=|A B|=50 \mathrm{~mm}$. 28 drain ports (with an inner diameter of each drain port equal to $0.6 \mathrm{~mm}$ ) were located over the middle cross section to determine the pressure coefficient $C_{p}$. Points $i=2-15$ were equidistant on the arc $A B$ with an angular spacing of $\pi / 15$ radians. Points $=16-29$ were placed on the straight section of $A B$ with a spacing of $L / 13$. Thin cutoff washers shaped as discs with a diameter $130 \mathrm{~mm}($ Fig. 1, c) were located at a distance of $d / 2$ to the left and to the right of the measuring section. They were intended to prevent the information exchange in terms of the semi-circular cylinder length.

The mean air flow velocity in the wind tunnel was $U=19.2 \mathrm{~m} / \mathrm{s}$. This corresponded to the Reynolds number Re $(=L U / v)=64000$ where $v$ is the kinematic viscosity coefficient. The turbulence in-

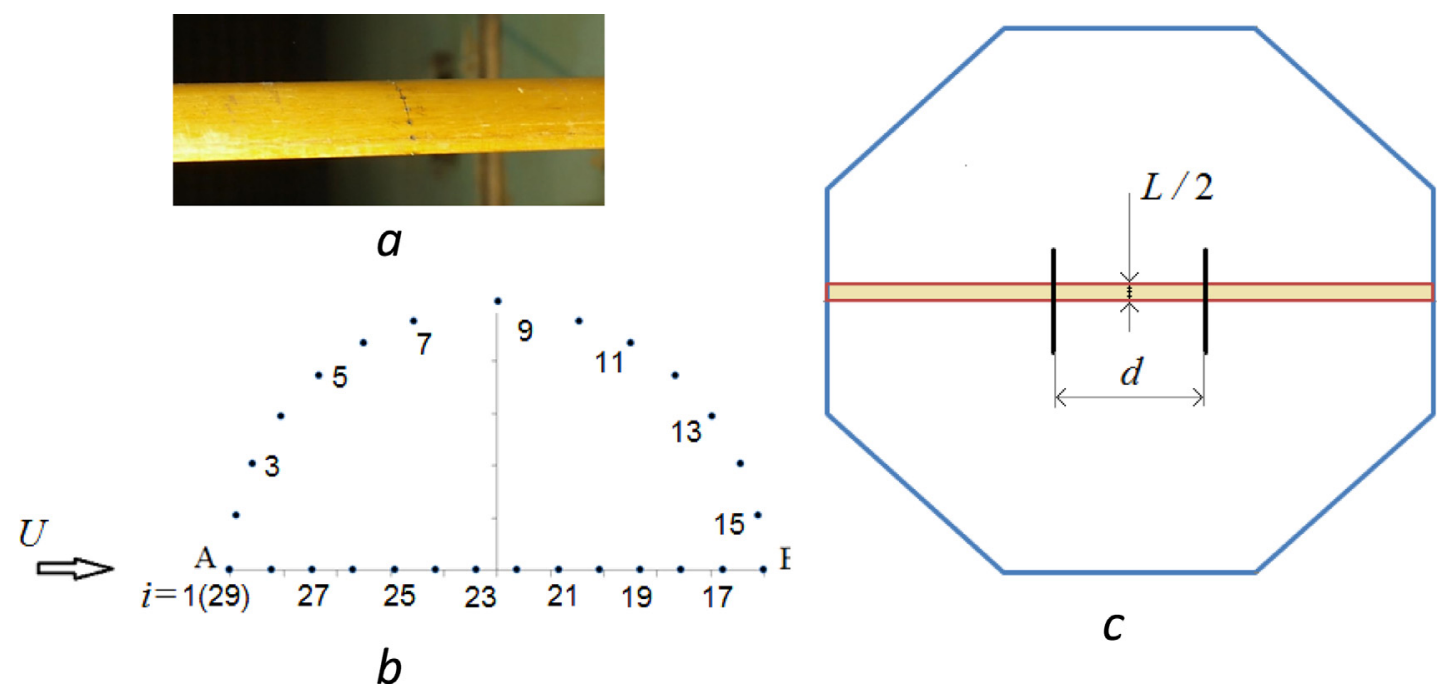

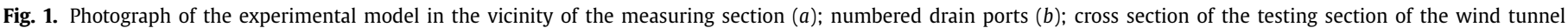

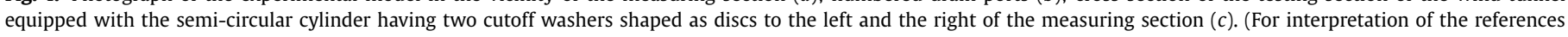
to colour in this figure legend, the reader is referred to the web version of this article.) 


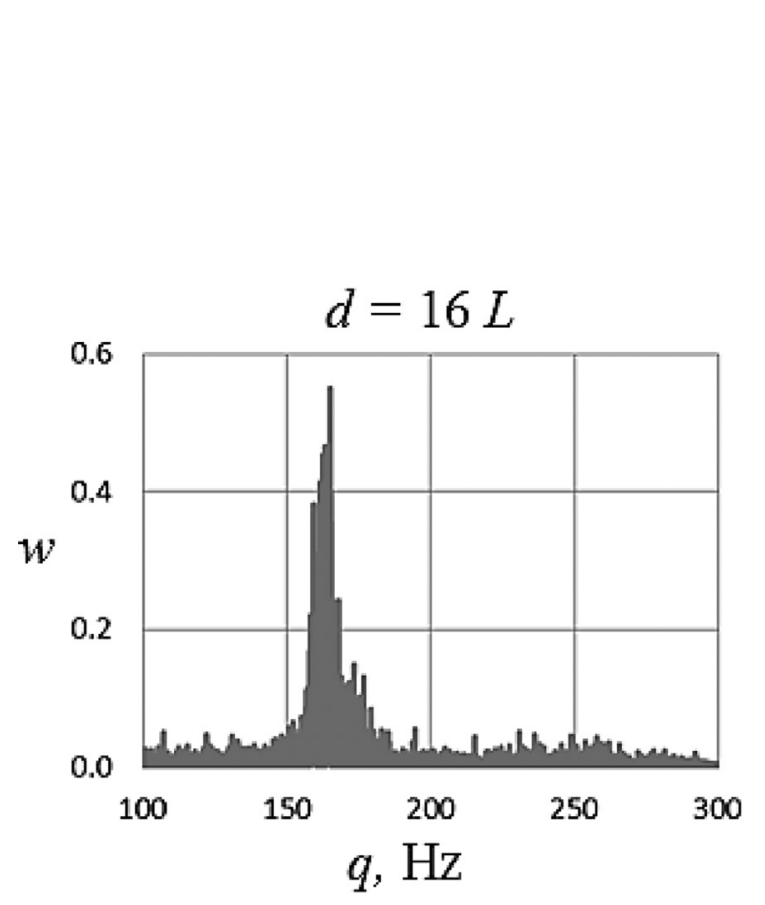

$a$

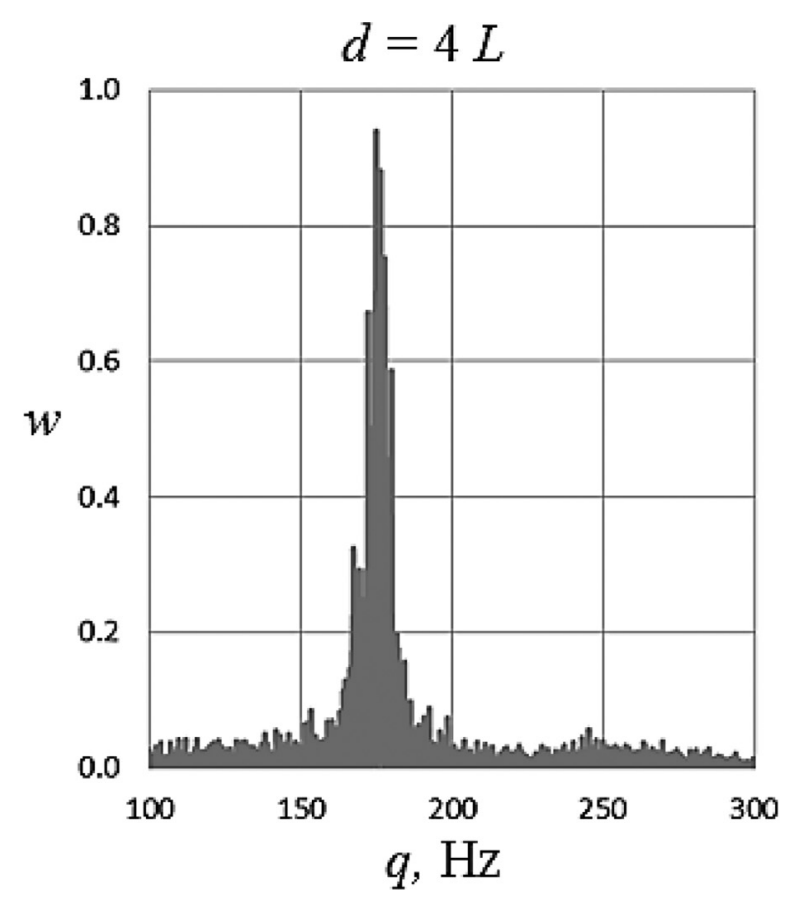

$b$

Fig. 2. Relative pulsation spectral density ( $w=\mathrm{PSD}_{10} \mathrm{~Pa}^{-} 2 \mathrm{~Hz}$ ) over the semi-circular cylinder surface at $i=12$.

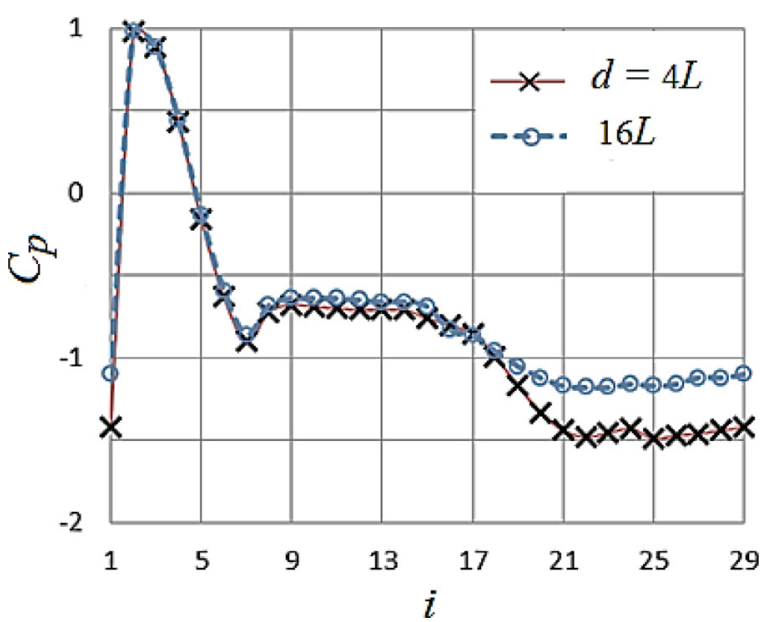

Fig. 3. Influence of cutoff washers on the time-averaged pressure coefficient distribution.

tensity of the undisturbed flow in the wind tunnel was $T u=0.4 \%$. Pressures at the semi-circular cylinder surface were measured using sensors MPXV7002 (mean relative error is 2.5\%) connected to a computer via an analog-to-digital converter $(A D C)$ with a sampling frequency of $1000 \mathrm{~Hz}$. The blockage of the wind tunnel cross section was less than $4 \%$; when computing $C_{p}$ the correction for the blockage effect was not therefore used. Integral drag $C_{x}$ and lift $C_{y}$ were computed in terms of $C_{p}$ distributions. Moreover, for the drain point $i=12$ the pulsation spectral density (PSD) was determined (Fig. 2). This spectrum has pronounced peaks responsible for the hydrodynamic frequency $\left(q=q_{g}\right)$ of shedding of large vortices from the semi-circular cylinder surface. The Strouhal number $\mathrm{Sh}=q_{g} L / U$ was determined in terms of this frequency.

Fig. 3 illustrates the computation results of distributions of the time-averaged pressure coefficients $C_{p}$ at two distances $d$ between the cutoff washers. It appeared that the approach of the cutoff
Table 1

Experimental estimates of the integral characteristics of averaged flow around the semi-circular profile at zero angle of attack.

\begin{tabular}{llll}
\hline Distance between cutoff washers & $C_{x}$ & $C_{y}$ & Sh \\
\hline $16 L$ & 0.467 & -0.634 & 0.410 \\
$4 L$ & 0.476 & -0.816 & 0.440 \\
\hline
\end{tabular}

washers practically did not affect the pressure distribution over the curvilinear surface of the semi-circular cylinder, but substantially (by 40\%) increased the rarefaction level on the straight section of $A B$. At that, the increase was seen both in the Strouhal number (by $7 \%$ ) and in the negative lift coefficient $C_{y}$ (Table 1 ).

A conclusion can be made that in this task, three-dimensional effects are mainly manifested themselves through the mechanism of information exchange in the direction of the semi-circular cylinder length in large-size separated flow regions. In these regions, this enables one to equalize the pressure and to decrease the rarefaction degree in comparison to two-dimensional flow. When cutoff washers are present, the above-mentioned three-dimensional mechanism can be substantially suppressed. Apparently, the experimental results on the pressure coefficient distribution over the semi-circular cylinder in the presence of cutoff washers at the distance $d=4 \mathrm{~L}$ are the best variant for comparison with twodimensional computations.

It should be noted that the independence of the obtained results on the Reynolds number was confirmed over the range $\operatorname{Re}=(4.9-7.3) \times 10^{4}$. The similar conclusion follows from the comparison of the data presented elsewhere in [1,2], In [2], integral force characteristics were directly measured by the weight method at $\operatorname{Re}=33000$. The obtained results appeared to be close to those at $\operatorname{Re}=67000$ [1]. This points to the fact that there is no Re influence in the given range. This can be a basis for substantiating the validity of using the experimental data at $\mathrm{Re}=64000$ to test the numerical result arrays obtained independently at $\mathrm{Re}=50000$. 


\section{Numerical simulation problem. Turbulence models}

The numerical study of turbulent incompressible flow around a semi-circular profile is performed with the consideration of a slight rounding of leading $A$ and trailing $B$ edges (Fig. 1). The chord length $L$ of the semi-circular profile is taken as the characteristic size so that the remaining linear sizes are expressed in terms of the chord. The rounding radius $R$ of the profile edges is varied from 0 to 0.005 . The angle of attack is assigned as equal to zero. Flow parameters at the computational domain inlet are taken as scales for nondimensionalization. The Reynolds number is assumed to be equal to 50000 .

As in [3,22], to solve the problem on two-dimensional unsteady turbulent incompressible viscous fluid flow around the semi-circular profile, the mathematical model based on the system of the unsteady Reynolds-averaged Navier-Stokes (URANS) equations is used.

To close the URANS system, several semi-empirical turbulence models of different complexity were used; most simple among them was the shear stress transport (SST) model that had found wide use for typical near-wall flows, including separated flows [18,19]. Menter's $k-\omega$ model generalizes two turbulence models: the $k-\varepsilon$ model of Launder and Spalding for shear flow regions far from the wall and the $k-\omega$ model of Saffman and Wilcox for the near-wall region. In addition, the ideas implemented in the turbulence model of Johnson and King were adopted to construct the zonal SST model. As previously mentioned, to determine eddy viscosity, the old SST model [18] uses the modulus of vorticity ' $\Omega$, whereas the new SST model [19], like most differentialtype semi-empirical models, includes the modulus of the tensor of strain rates $S$ into the expression for eddy viscosity. It is of importance to emphasize that the semi-empirical models are mainly validated on the basis of near-wall flow results; the necessity then appears to validate them using separated flow data. As noted in [20], the Rodi-Leschziner approach with the eddy viscosity correction, widely used in the late $80 \mathrm{~s}$ of the last century [30] in the high-Reynolds version of the two-parameter dissipation turbulence model of Launder and Spalding, says that eddy viscosity is affected by the correction function $f_{c}=1 /\left(1+C_{c} \times \mathrm{Ri}_{t}\right)$; in this case, the constant $C_{C}=0.57$ is defined analytically when computing turbulent annular and twin parallel jets [31] and the constraint is set on the product $f_{c} \times C_{\mu}: 0.02<f_{c} \times C_{\mu}<0.15$ (in the standard $k-\varepsilon$ model [30], the semi-empirical constant $C_{\mu}=0.09$ in the eddy viscosity expression). S. Isaev generalized the Rody-Leschziner approach to Menter's model [19]. Kharchenko, Usachov, and Isaev chosen the constant $C_{C}=0.02[20]$ from the condition of the best agreement between numerical predictions and experimental data for numerous examples of separated flows. Not so long ago, Smirnov and Menter [23] proposed one more correction (SM correction) of the SST model [19] by extending the Shur-Spalart correction in the eddy-viscosity model of Spalart and Allmaras [24] to Menter's two-parameter model. Durbin's correction introduced into the SST model [19] should be mentioned.

The near-wall conditions for SST models are formulated from the condition that the normal derivative to the wall is equal to zero for turbulence energy and the specific dissipation rate of turbulence energy is defined according to [32].

The two four-parameter $\zeta-f$ and $\zeta-a$ models are considered as semi-empirical models of high complexity. As previously mentioned, the $\zeta-f$ model was developed by Hanjalić [26] and is fairly well known. Therefore, the recently developed $\zeta-a$ model will be more detailed. It was built by reducing the Reynolds stress transport model with elliptic blending [27,28] to the four-parameter eddy-viscosity model. In fact, this model is the version of Durbin's original model [33] based on the following eddy-viscosity depen- dence valid in the vicinity of the wall

$\mu_{t}=\rho C_{\mu} \overline{v^{2}} T_{t}$,

where $\overline{v^{2}} \equiv \overline{u^{\prime}{ }_{2} u_{2}^{\prime}}$ is the Reynolds stress tensor component for velocity fluctuations normal to the wall; subscript 2 stands for the direction normal to the wall; the constant $C_{\mu}=0.21 ; T_{t}$ is the turbulence time scale; $\rho$ is the medium density.

From the system of the equations of the Reynolds stress transport model we selected one transport equation of the Reynolds stress tensor component for velocity fluctuations normal to the wall:

$\rho \frac{D \overline{v^{2}}}{D t}=D_{22}+\rho\left(\phi_{22}-\varepsilon_{22}\right)$.

The right hand-side of Eq. (3.1) sequentially contains the diffusion term, the re-distribution term, which represents a correlation between pressure and strain rate tensor, and the dissipation rate term. In the near-wall layer, the production of the Reynolds stress tensor component normal to the wall is absent; to model the diffusion term the gradient hypothesis is adopted:

$D_{22}=\nabla \cdot\left[\left(\mu+\mu_{t}\right) \nabla \overline{v^{2}}\right]$.

By analogy with the $\zeta-f$ model [26], we pass to a dimensionless variable $\zeta=\overline{v^{2}} / k$

$\frac{D \zeta}{D t}=\frac{1}{k} \frac{\overline{v^{2}}}{D t}-\frac{\overline{v^{2}}}{k^{2}} \frac{D k}{D t}$,

where $k$ is the turbulence kinetic energy. Using the transport equation of turbulence kinetic energy

$\rho \frac{D k}{D t}=\nabla \cdot\left[\left(\mu+\frac{\mu_{t}}{\sigma_{k}}\right) \nabla k\right]+P-\rho \varepsilon$

and Eq. (3.1), the transport equation for $\zeta$ assumes the form:

$\rho \frac{D \zeta}{D t}=\nabla \cdot\left[\left(\mu+\frac{\mu_{t}}{\sigma_{k}}\right) \nabla \zeta\right]+\rho \frac{1}{k}\left(\phi_{22}-\varepsilon_{22}\right)-\frac{\zeta}{k}(P-\rho \varepsilon)+X$,

where the cross-diffusion term can be written as:

$X=\frac{2}{T_{t} \varepsilon}\left(\mu+\frac{\mu_{t}}{\sigma_{k}}\right)(\nabla \zeta \cdot \nabla k)$.

Here $\varepsilon$ is the isotropic dissipation rate of turbulence kinetic energy, $P$ is the turbulence kinetic energy production calculated as $P=\mu_{t} 2 S_{i j} S_{i j}$, and $S_{i j}$ is the strain rate tensor.

Having re-arranged the variables in Eq. (3.3) and having introduced the notation

$f \equiv \frac{1}{k}\left(\phi_{22}-\varepsilon_{22}+\varepsilon \zeta\right)$,

we have the transport equation in the following form:

$\rho \frac{D \zeta}{D t}=\nabla \cdot\left[\left(\mu+\frac{\mu_{t}}{\sigma_{k}}\right) \nabla \zeta\right]+\rho f-\frac{\zeta}{k} P+X$.

The similar equation, but with the discarded cross-diffusion term is formulated in the $\zeta-f$ model [26]. According to the initial model based on the blending function [28], the function $f$ in the transport equation for $\zeta$ is calculated using the blending function $a$ :

$f=\left(1-a^{3}\right) f_{w}+a^{3} f_{h}$,

where the value of the function $f$ near the wall is

$f_{w}=\frac{1}{k}\left(\phi_{22}^{w}-\varepsilon_{22}^{w}\right)+\frac{\varepsilon}{k} \zeta=-6 \frac{\varepsilon}{k} \zeta+\frac{\varepsilon}{k} \zeta=-5 \frac{\varepsilon}{k} \zeta$

and the value of the function $f$ far from the wall is

$f_{h}=\frac{1}{k}\left(\phi_{22}^{h}-\varepsilon_{22}^{h}\right)+\frac{\varepsilon}{k} \zeta=-\frac{\varepsilon}{k}\left(C_{1 f}-1+C_{2 f} \frac{P}{\rho \varepsilon}\right)\left(\zeta-\frac{2}{3}\right)$. 
The model constants are taken from the basic second-moment model [28] and are based on the model by Speziale, Sarkar and Gatski (SSG) [29]: $\sigma_{k}=1.0, \quad C_{1 f}=1.7, \quad C_{2 f}=0.9$.

The equation for the elliptic blending function $a$ is formulated as follows:

$\nabla^{2} a+\frac{1}{L_{t}^{2}}(1-a)=0$

where the scale $L_{t}$ is calculated from the realizability conditions and is bounded from below by Kolmogorov's length scale:

$$
\begin{gathered}
L_{t}=C_{L} \max \left[\min \left(\frac{k^{3 / 2}}{\varepsilon}, \frac{k^{1 / 2}}{C_{\mu} \sqrt{6 S_{i j} S_{i j}} \zeta}\right), c_{\eta}\left(\frac{\mu^{3}}{\rho^{3} \varepsilon}\right)^{1 / 4}\right], \\
c_{\eta}=80, \quad C_{L}=0.133
\end{gathered}
$$

The realizability conditions $2 k \geq \overline{u^{\prime}{ }^{2}} \geq 0$ [26] are used for a relationship between the turbulent stress tensor and the strain rate tensor expressed in the Boussinesq hypothesis as $\rho \overline{u_{i}^{\prime} u^{\prime}{ }_{j}}=$ $-2 \mu_{t} S_{i j}+2 / 3 k \delta_{i j}$. As a result, the restrictions on the turbulence scales of length (3.9) and time (3.11) are formulated.

The transport equation for the dissipation rate of turbulence kinetic energy is taken from the basic model:

$\rho \frac{D \varepsilon}{D t}=\nabla \cdot\left[\left(\mu+\frac{\mu_{t}}{\sigma_{\varepsilon}}\right) \nabla \varepsilon\right]+\frac{C_{\varepsilon 1} P-C_{\varepsilon 2} \rho \varepsilon}{T_{t}}$,

where the model constants are:

$$
\begin{aligned}
& C_{\varepsilon 1}^{\prime}=C_{\varepsilon 1}\left(1+A_{2}\left(1-a^{3}\right) \frac{P}{\rho \varepsilon}\right), \quad A_{2}=0.065, \quad \sigma_{\varepsilon}=1.3, \\
& C_{\varepsilon 2}=1.83, \quad C_{1 f}=1.44
\end{aligned}
$$

and the turbulence time scale with the imposed realizability conditions are:

$T_{t}=\max \left[\min \left(\frac{k}{\varepsilon}, \frac{0.6}{C_{\mu} \sqrt{6 S_{i j} S_{i j}} \zeta}\right), c_{T}\left(\frac{\mu}{\rho \varepsilon}\right)^{1 / 2}\right]$.

To determine the production coefficient in the near-wall layer, it is possible to use the alternative formula [28]:

$C_{\varepsilon 1}^{\prime}=C_{\varepsilon 1}\left(1+A_{1}\left(1-a^{3}\right) \frac{1}{\sqrt{\zeta}}\right), A_{1}=0.03$

As in all models of this family, turbulent viscosity is determined by the formula:

$\mu_{t}=\rho C_{\mu} k \zeta T_{t}, \quad C_{\mu}=0.21$

Wall boundary conditions when integrating up to the wall are set as follows:

$k=0, \varepsilon=2 \mu k / \rho y^{2}, \zeta=0, a=0$

The model can be used with arbitrary wall functions. Here, as in the case of the turbulence kinetic energy, for the dimensionless quantity $\zeta$ the condition of no diffusion flux through the wall: $\partial \zeta / \partial n=0$ can be used.

The wall boundary condition for the elliptic blending function $a$ is determined from the calculations of the developed turbulent channel flow and is the function of the dimensionless distance, $y_{1}^{+}$, from the wall to the first near-wall node:

$a_{W}=1-\exp \left(-\frac{y_{1}^{+}}{A^{+}}\right), \quad A^{+} \sim 18$.

In the viscous sublayer, the value of $a$ tends to zero.

Finally, the model is described by a set of the differential Eqs. $(3.2,3.4,3.8,3.10)$ and by the expressions for turbulence quantities $(3.9,3.11,3.12)$.

At present, a great number of models based on Durbin's model, including the models with the strategy of the elliptic blending function, have been developed. The most comprehensive analysis of the models is presented in [34]. The result strongly depends on the model version and can be substantially different from experimental data. The predictive efficiency of models is mainly affected by model details, chosen constants, and boundary conditions not relating directly to the basic concept of Durbin's model.

Undisturbed flow parameters are assigned at the computational domain inlet. Turbulence characteristics are determined in the same manner as in $[3,10,15,22]$ for the conditions of wind tunnel experiments. So, the turbulence energy $k_{\infty}$ at the domain inlet is assigned in terms of the incoming flow turbulence intensity $T u_{\infty}=1.5 \%$ and the turbulence scale $l_{\infty}$ is chosen from the condition when eddy viscosity is close to physical one.

Outflow boundary conditions (conditions for continuation of solution) are set at the computational domain outlet, whereas no-slip conditions - at the body surface. Near the wall, $y+$ does not exceed 1. The state of collision of a uniform flow with an abruptly decelerated thick body is taken as the initial condition.

\section{CFD codes. Computation methodology}

Based on solving the unsteady Reynolds-averaged Navier-Stokes with the use of semi-empirical models, applied software became a powerful tool to predict flow parameters and turbulence characteristics. In the present study, by the example of solving the test two-dimensional task for incompressible viscous turbulent fluid flow around a semi-circular profile the commercial industrial Fluent [35], CFX [36], StarCCM+ [37], and OpenFOAM codes [38], as well as research VP2/3 [15] and SigmaFlow [39] codes were compared.

All computational algorithms realized in these codes are based on both the concept of physical-operator splitting and grid methods for solution of the governing equations [11,15], The use of this concept allows the system of partial differential equations to be divided into blocks containing momentum equations in natural variables (including the Cartesian velocity components for incompressible viscous fluid flow), pressure correction equation (SIMPLEC [40,41]] replacing continuity equation, and equations for their closure (from the selected turbulence model) [15]

Thus, the system of the unsteady-state equations transformed into discrete form is being solved block by block at each time step in the course of the global iteration process (about 10-20 iterations), when for one iteration in the course of solving the momentum equations, several (about 10-15) iterations are being performed in the pressure correction block and about 4-6 iterations in the turbulence block.

The equations are either immediately written in discrete form (Fluent, CFX, StarCCM+, and OpenFOAM codes [41]]), or are preliminarily linearized (SigmaFlow and VP2/3 codes [15,42]).

In the present study, the VP2/3 code is selected as the basic one and used for testing computations (together with other codes). The specific features of the used algorithm for this code are: (1) the pressure correction procedure SIMPLEC [40] based on the concept of physical-operator splitting together with the generalized RhieChow monotone approximation for a given centered computational block [43,44]; (2) the approximation of the convective terms in the explicit hand-side of the momentum equations by the onedimensional analog of Leonard's quadratic upwind scheme (QUICK) [45] to reduce the influence of numerical diffusion specific for the considered type of separated flows and by Van Leer's scheme [46] for the equations for turbulence characteristics; (3) the representation of the convective terms in the implicit hand-side of the momentum equations by means of the upwind scheme (QUICK) with one-sided differences, which allows the stability of the computational procedure to be improved; 4) the application of the methods with preconditioners for solution of difference equations [47] 
Table 2

Used codes, temporal and spatial discretization schemes, solvers.

\begin{tabular}{|c|c|c|c|c|c|}
\hline Code & $\begin{array}{l}\text { Equation form: } \\
\text { incremental (delta) } \\
\text { form/usual (delta) form }\end{array}$ & $\begin{array}{l}\text { Methods for global } \\
\text { iteration }\end{array}$ & $\begin{array}{l}\text { Discretization of } \\
\text { convective terms }\end{array}$ & $\begin{array}{l}\text { Time discretization } \\
\text { scheme }\end{array}$ & Solver for algebraic equations \\
\hline $\mathrm{VP} 2 / 3$ & Delta form $[14,44]$ & SIMPLEC [42] & $\begin{array}{l}\text { QUICK- scheme for } \\
\text { momentum } \\
\text { equations and } \\
\text { TVD-scheme with } \\
\text { Van Leer limiter for } \\
\text { turbulence equations } \\
{[47,48]}\end{array}$ & $\begin{array}{l}\text { Second Order Upwind } \\
\text { Euler (SOUE) scheme } \\
{[50]}\end{array}$ & $\begin{array}{l}\text { Fixed-point iteration with AMG } \\
\text { preconditioner for pressure } \\
\text { correction and ILU }(0) \\
\text { preconditioner for another } \\
\text { variables }[49]\end{array}$ \\
\hline SigmaFlow & Delta form & SIMPLEC & $\begin{array}{l}\text { QUICK- scheme for } \\
\text { momentum } \\
\text { equations and UMIST } \\
\text { TVD-scheme for } \\
\text { turbulence equations }\end{array}$ & SOUE scheme & $\begin{array}{l}\text { BiCGSTAB with AMG } \\
\text { preconditioner from } \\
\text { Demidov's library (amgcl) for } \\
\text { pressure corrector and DILU } \\
\text { for another variables }\end{array}$ \\
\hline Fluent & Usual form [10] & $\begin{array}{l}\text { SIMPLEC Face pressure } \\
\text { is obtained from the } \\
\text { linear law }\end{array}$ & $\begin{array}{l}\text { QUICK-scheme with } \\
\text { min/max limiter }\end{array}$ & SOUE scheme & AMG for all variables \\
\hline CFX & Usual form & Coupled [52] & $\begin{array}{l}\text { TVD-scheme with } \\
\text { limiter [53] }\end{array}$ & SOUE scheme & AMG for all variables \\
\hline Star $\mathrm{CCM}+$ & Usual form & PISO [10] & $\begin{array}{l}\text { TVD-scheme with } \\
\text { min/max limiter [51] }\end{array}$ & $\begin{array}{l}\text { Euler first-order } \\
\text { scheme }\end{array}$ & $\begin{array}{l}\text { AMG for all variables. For } \\
\text { pressure correction, the } \\
\text { preconditioned conjugate } \\
\text { gradient method is used [52] }\end{array}$ \\
\hline Open FOAM & Usual form & PISO [43] & $\begin{array}{l}\text { TVD-scheme with Van } \\
\text { Leer limiter }[48]\end{array}$ & $\begin{array}{l}\text { Euler first-order } \\
\text { equation }\end{array}$ & $\begin{array}{l}\text { AMG for pressure and } \\
\text { biconjugate gradient solver } \\
\text { (BiCG) with ILU } \\
\text { preconditioner for another } \\
\text { variables [49] }\end{array}$ \\
\hline
\end{tabular}

The use of the generalized Rhie-Chow approach [44] permitted one to avoid difficulties in calculating unsteady flows. The flows calculated by means of the generalized Rhie-Chow monotone approximation are used not only in the pressure correction equation, but are remembered and taken to calculate the convective terms of all transport equations (turbulence). The numerical procedure is thus supplemented by one more stage - calculation of flows at cell edges. It is performed after solving the momentum equation before the pressure correction stage. After the pressure correction has been determined, not only velocities in the cell centers, but also normal velocity components at the cell edges, i.e., mass flow values, are corrected. To determine a normal velocity component value at a cell edge, not simple averaging of velocity values, as in [42], but averaging of velocity values expressed from the discrete form of the writing of the momentum equation in adjacent cells is used. When determining a normal component of a velocity at a cell edge, it becomes possible to identify components proportional to this velocity at the previous iteration (previous time step), to assign a velocity dependence on mass forces, and to introduce into the velocity definition a local pressure gradient normal component value calculated only in terms of velocity values in adjacent cells. As a matter of fact, the generalized Rhie-Chow monotone approximation in this approach appears automatically with the coefficient equal to the relaxation coefficient of the momentum equation. Since the current flow value at the cell edge contains the previous iteration (time step) value, the changes in these parameters do not sharply increase errors.

The time derivatives in the differential equations are discretized according to Euler's method [11,15] and Second Order Upwind Euler SOUE-scheme [48],

The method for solution of algebraic equations is the BiCGSTAB preconditioner [47] with the AMG preconditioner from Demidov's library (amgl) [49] for pressure correction and the ILU(0) preconditioner for another variables.

The multiblock computational technology (MCT) realized in the VP2/3 code is outlined elsewhere in [14,15], The essence of this technology is to introduce a set of different-scale, tier, and overlapping structured grids matched with possible specific flow regions.
In the two rows of near-boundary cells of each of the overlapping or overset grids, the parameters are determined at linear interpolation [15,16] in the manner, as done in [13], In [15], it is shown that this approach is equivalent to the application of adaptive unstructured grids, but its computational resources are substantially less, i.e., it is more efficient. It also provides a proper computational accuracy without refining grids, since it automatically resolves the flow structure.

Computation from grid to grid according to MCT at linear interpolation is a source of errors; however, the testing computations of steady circulation flow in a cavity with a moving cover [16] show that the errors appear to be quite acceptable. Nevertheless, to assess the computational accuracy of unsteady turbulent flows, we performed testing computations of flow around the semi-circular profile on the composite grids obtained from the overlapping structured grids by eliminating the overlapping zones of grids and replacing them by unstructured grids consisting of fragments. The algorithms realized in the VP2/3 code allow one to solve tasks on such unstructured grids having 90\% of structured fine grids. It should be noted that the composite grids are the analogs of "DRAGON"-type grids [12], but they serve not so much to describe flows in multiply connected domains as to correctly resolve the flow structure and the physical processes in the task at hand. Table 2 summarizes the specific features of algorithms and computational methods realized in SigmaFlow, VP2/3, Fluent, CFX, StarCCM+, and OpenFOAM codes.

\section{Testing studies}

Testing studies cover a wide range of testing computations of periodic incompressible fluid flow around a semi-circular profile at zero angle of attack. We consider how solutions are affected by scheme factors, in particular computational grids, time and nearwall steps, numerical discretization schemes of convective and time terms of equations, and edge rounding. The MCT accuracy is assessed. Various semi-empirical turbulence models are compared, and the advantage of the SST model modified within the RLI ap- 

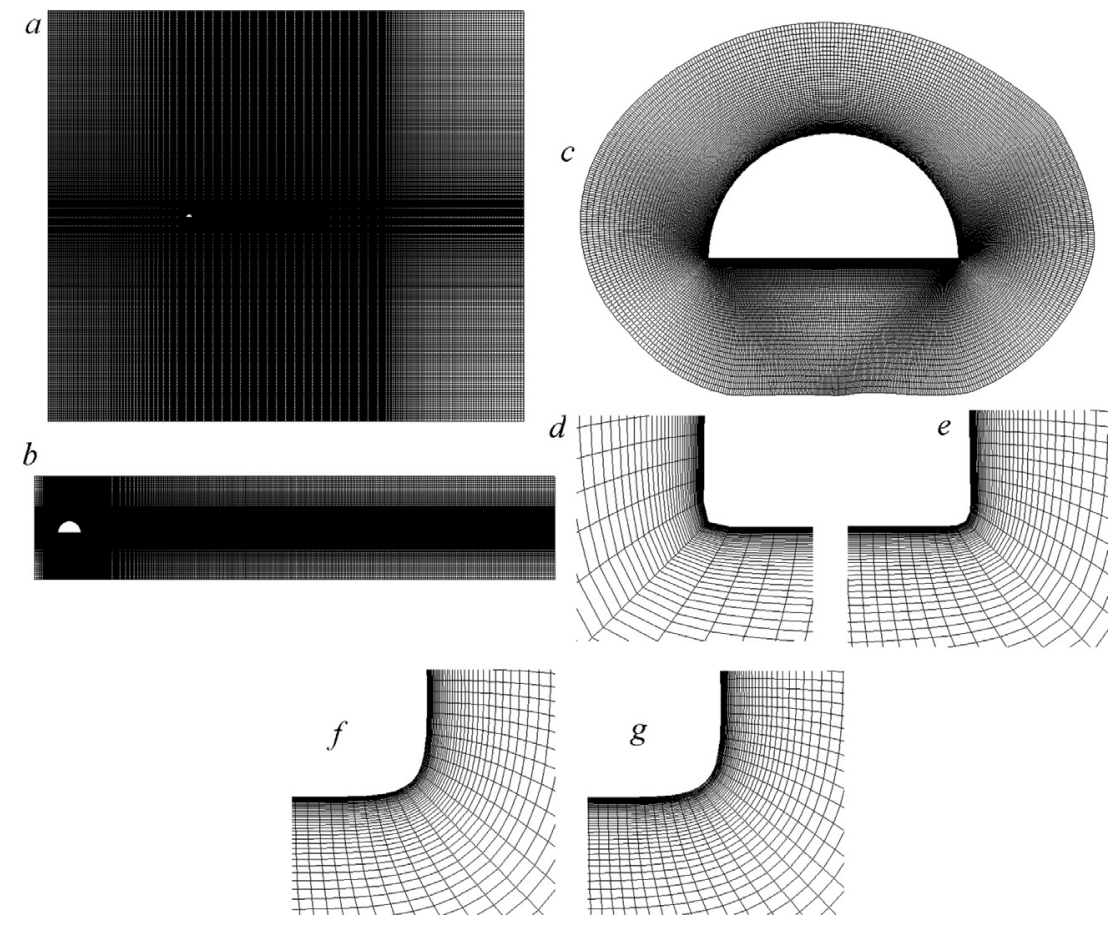

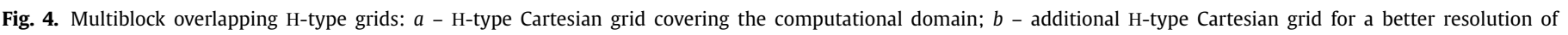

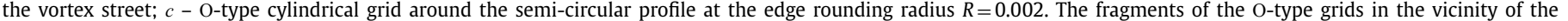
semi-circular profile rounded leading $(d)$ and trailing $(e, f, g)$ edges at different rounding radii: $d, e-R=0.002 ; f-0.003 ; g-0.005$.

proach is shown. The computation results obtained by the chosen software are compared.

\subsection{Grids and their classification}

The grids built to compute the test problem of turbulent flow around the semi-circular profile at zero angle of attack mainly use the experience of solving the problem of flow around an NACA0012 airfoil with an angle of attack ranging from $0^{\circ}$ to $180^{\circ}$ [10], When building a grid, it is first important to move away inlet and outlet, upper and lower boundaries of a computational domain far from a profile so that the defined boundary conditions will have no influence on flow near a body and in its wake. It is also of significance to properly refine a grid into cells in the vicinity of the profile in order to correctly resolve the flow structure: thin boundary layer, shear layers at the separated flow boundaries, as well as a vortex street developing behind the profile. With MCT used, this is done by introducing an automated connection of overlapping fragmentcomposed grids close to simple-topology orthogonal grids meant to resolve the flow structure.

Several-type computational grids are designed for the basic VP2/3 code. The simplest multiblock O-type grid consists of two tiers: O-type grids extending to the computational domain outlet and O-type grids around the profile. The semi-circular profile edges are rounded at the radius $R=0.005$. The center of the Cartesian $x$, $y$ coordinate system is located in the center of the profile base. The grid is the analog of the O-type grid [3,22] with a decreased nearwall grid step equal to $10^{-5}$. The number of O-type grid cells surrounding the profile in the vertical direction is 50 . The size of the near-wall zone is 0.05 . 420 cells are located on the top arch of the profile. The minimal grid step in the near-edge zone is $5 \times 10^{-4}$. The total number of the cells of the grid adjacent to the profile is equal to 33228, among which are 32000 computational cells. As a result, 640 cells are obtained in the direction to the ring.

The radius of the outer O-type grid of the computational domain is equal to 42.6. In the direction to the ring, the domain is divided into 420 cells. In the radial direction, there are two areas: the length of the first area is 7 chords and the second - 35 chords. The both areas contain 160 cells each; the grid step at the end of the first area is equal to 0.2. The outer O-type grid contains 134918 cells, among which are 134400 computational cells. Finally, the multiblock O-type grid is divided into 166400 computational cells. It can be characterized as a rather coarse grid, especially in the far vortex wake. It is designated as 0 .

For O-type grids, it is necessary to set fixed boundary conditions at the inlet of the outer grid (one half the ring on the left side, in the incoming flow oriented from left to right) and outflow boundary conditions at the computational domain outlet (one half the ring boundary, through which a far vortex wake flows). Not always, a transition from boundary conditions of one type to those of another favorably affects the computational process.

The SST model with the correction within the RLI approach and with the constant $C_{C}=0.02$ is used. The problem is solved using the second-order approximation at a time step of 0.01 . The number of iterations in the pressure correction block is 6 and the number of iterations at each time step - 30. The distribution of characteristics calculated at $\operatorname{Re}=40000$ is taken as the initial approximation. The process of solving the problem is carried out to the moment of reaching the self-oscillatory period with close maximum and minimum values of $R_{x}$ and $R_{y}$. It should be noted that when solving the problem the errors of dependent variables show their periodic change.

The $R_{x}$ and $R_{y}$ distributions on the self-oscillatory period, the surface distribution of the pressure coefficient $C_{p}$, as well as the averaged flow field patterns and the local extreme characteristics will be compared by analyzing the results of flow computed on different grids.

Fig. 4 illustrates several H-type overlapping grids. Fig. 4, b demonstrates the variant of a longer and finer grid in the wake. The profile edges are rounded at $R=0.002-0.005$ (Fig. 4 $d-g$ ). The center of the Cartesian coordinate system is located in the center of the profile base. 
A multiblock grid consists of three fragments: a O-type grid around the profile (Fig. 4,c) and two H-type grids, one of which covers the computational domain outlet, and another additional grid is meant to resolve in detail a vortex street developing in the near and far wakes behind the profile.

The near-wall step of the O-type grid is equal to $10^{-5}$. The total number of grid cells in the vertical direction to the semi-circular profile is 112 . The size of the near-wall zone is 0.5 . 580 cells are located on the profile. The minimal grid step in the leading nearedge zone is $2 \times 10^{-3}$ and in the trailing one $-10^{-3}$ (Fig. 4 d,e). The grid step in the middle of the top arch of the profile is $10^{-2}$. To match the additional rectangular grid, into which the circular grid adjacent to the profile is placed, the near-boundary step in the radial direction is equal to 0.025 . Thus, the fragment-composed O-type grid contains 64380 cells, among which are 63220 computational cells.

The outer H-type grid around the entire computational domain extends from $x=-21.5$ to $x=51.2$ and from $y=-31.05$ to $y=31.44$. Minimal grid steps equal to 0.1 in the both directions are located in the vicinity of the profile. The maximal step near the right computational domain boundary does not exceed 0.5 . The total number of fragment-composed H-type grid cells is 180873 .

To improve the computational accuracy of the vortex street, the additional H-type grid extends from $x=-1.5$ to $x=21$ and from $y=-2.05$ to $y=2.44$. Minimal grid steps in the both directions are equal to 0.025 . The total number of grid cells is 54634, among which are 52326 computational cells.

The total number of computational cells of the grid is about 300 thousands. It is designated as Ho.

At the edge rounding radius $R=0.003$, the near-edge grids nearby are similar in shape and size (Fig. 4f). The near-wall grid step is equal to $10^{-5}$. The number of cells in the vertical direction to the grid adjacent to the profile is 112 . The size of the near-wall zone is 0.5 . 856 cells are located on the profile. The minimal grid step in the leading and trailing near-edge zones is $4 \times 10^{-4}$. The grid step in the middle of the top arch of the profile is $10^{-2}$. To match the additional rectangular grid, into which the circular grid is placed, the near-boundary step in the radial direction is equal to 0.025 . Thus, the fragment-composed grid contains 95016 cells, among which are 93304 computational cells.

The multiblock grid at the edge rounding radius $R=0.003$ is designated as $\mathrm{Hr}$.

At the edge rounding radius $R=0.005$, the near-edge grids nearby are similar in shape and size (Fig. $4, \mathrm{~g}$ ). The near-wall grid step is equal to $10^{-5}$. The number of cells in the vertical direction to the grid adjacent to the profile is 112 . The near-wall zone size is 0.5 . 806 cells are located on the profile. The minimal step in the leading and trailing near-wall zones is $5 \times 10^{-4}$.The grid step in the middle of the top arch of the profile is $10^{-2}$. To match the additional grid, into which the circular grid is placed, the near-boundary grid step in the radial direction is 0.025 . Thus, the fragment-composed O-type grid contains 89577 cells, among which are 87963 computational cells.

The multiblock grid with the edge rounding radius $R=0.005$ is designated as $\mathrm{H}^{*}$.

Thus, the number of multiblock H-type grid cells in the two last cases increases by about 30 thousands and is approx. 330 thousand cells.

To analyze the testing computations using H-type grids meant to assess the influence of the time approximation of different order, as well as the size change and the grid fragment discretization, several rather coarse grids of the same topology as $\mathrm{H}-, \mathrm{Hr}-$, and $\mathrm{H}^{*}-$ grids are considered.

The near-wall step of the first of such grids designated as H1 is coarse and amounts to $10^{-4}$.

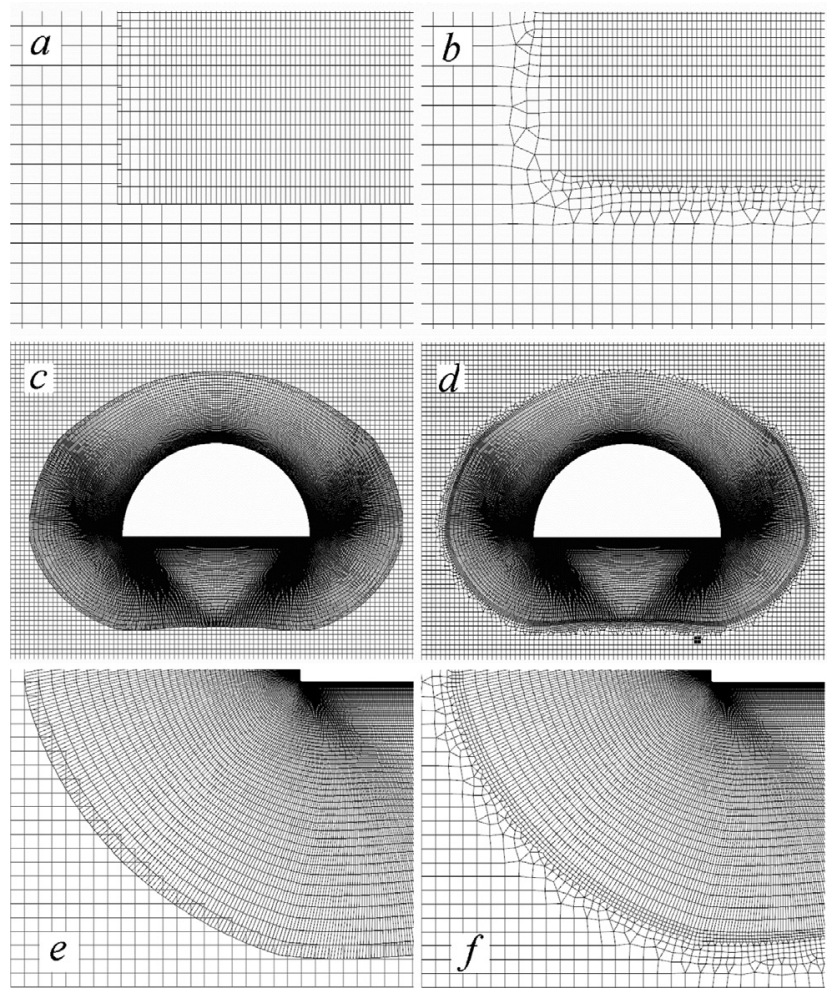

Fig. 5. Fragments of the multiblock structured $\mathrm{H}^{*}$-type grids $(a, c, e)$ and the relevant unstructured fragments of the designed composite grid $(b, d, f): a, b$ - insertion of the additional grid for wake computation into the outer Cartesian grid; $c, d$ - connection between the O-type grid surrounding the semi-circular profile and the additional rectangular grid for wake computation and $e, f$ - connection (enlarged scale).

The next multiblock H-type grid is designated as H1a, and the near-wall grid step decreases in comparison to the H1-type grid and is equal to $10^{-5}$.

The composite $\mathrm{H}^{*} \mathrm{k}$-type grid is based on the $\mathrm{H}^{*}$-type one. Fig. 5 shows some stages of transforming a multiblock grid into a composite one. The overlapping zones between the grid for wake computation and the outer grid, as well as between the near-wall grid and the grid for wake computation are replaced by unstructured fragments. To do this, a computational domain of a transverse size approximately equal to a four-fold maximal grid step in the overlapping zone of grids is first cut from the overlapping grids and is then replaced by an unstructured fragment.

Problems are solved on H-type grids by means of the first- and second-order approximations at a time step of 0.01 . The number of iterations in the pressure correction block is 10 and the number of iterations at each time step - 25. The distribution of the characteristics corresponding to the collision conditions, i.e., to an abrupt deceleration of the profile moving with a flow velocity is assigned as the initial approximation. The process of solving the problem is performed to the moment of achieving the self-oscillatory regime with close maximum and minimum values of $R_{x}$ and $R_{y}$. It should be noted that when solving the problem the errors obtained formulate their periodic change.

Computations are made on two grids implemented in the SigmaFlow and Fluent codes. The first of them is designated as MF.

The chord length is equal to 1 . The computational domain height is 40 , the length is 20 , the width in the transverse direction is 1 (the grid is three-dimensional and one cell is assigned in the transverse direction). A streamlined profile is a semi-circular profile without the rounding of its edges. An unstructured grid consisting of hexahedral cells is generated in ICEM. The grid has three fragments (Fig. 6,a,b): outer region (1), inner region (2), near-wall layer. The grid domain contains cells of about 0.2 . The inner region 

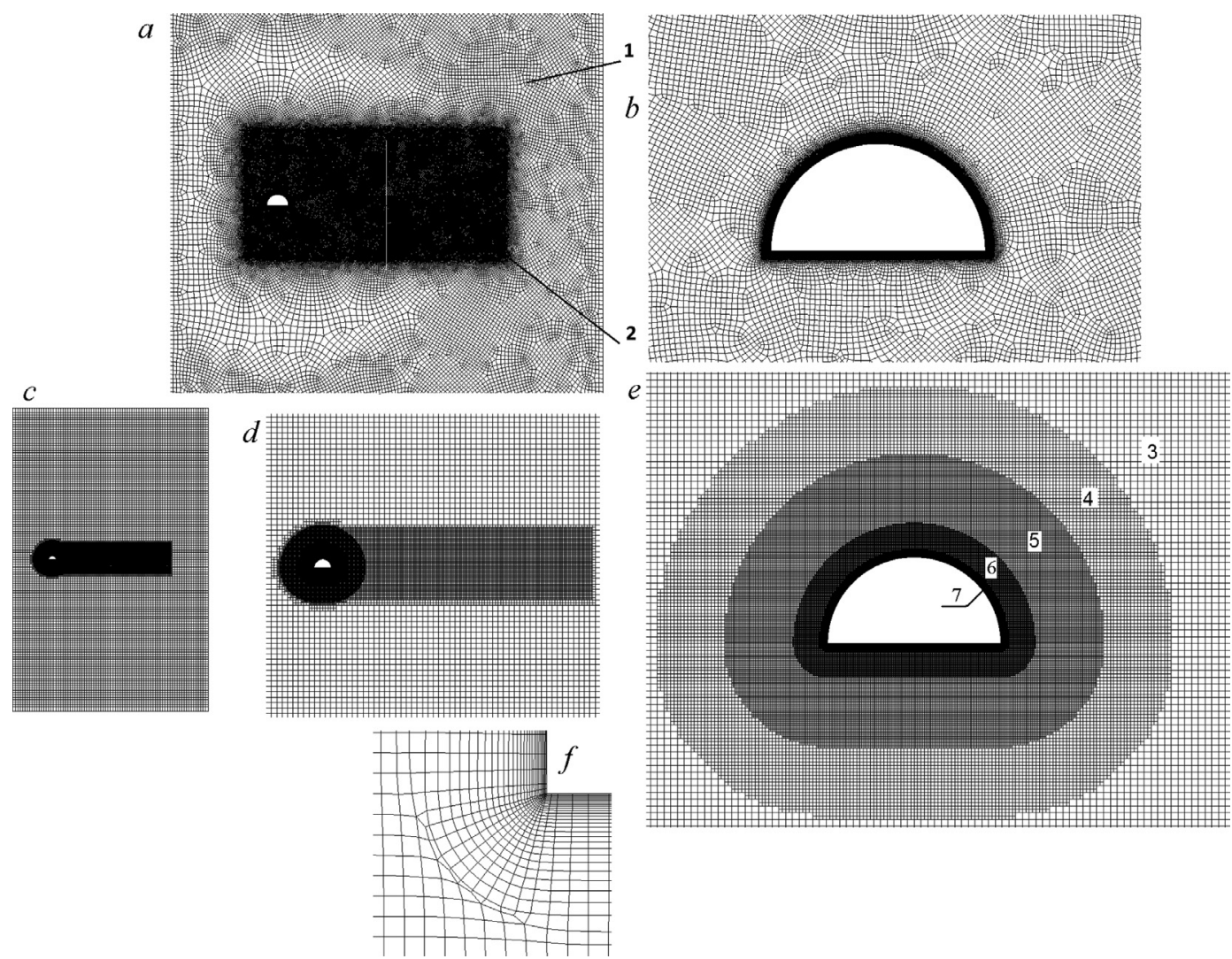

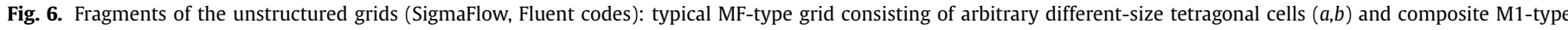

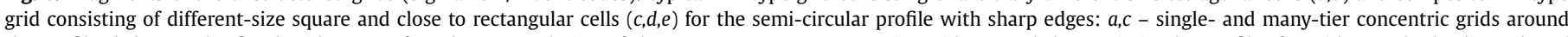

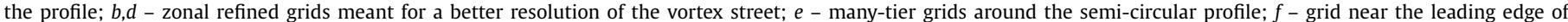
the profile. 1,2 - outer and refined grids meant for resolution of the vortex street; $3-7$ - refined tier grids.

Table 3

Parameters of M1-type grid layers.

\begin{tabular}{lll}
\hline & $\begin{array}{c}\text { Distance to the external boundary of the computational } \\
\text { domain }\end{array}$ & Grid step \\
\hline 1 & 0.05 & 0.00245 \\
2 & 0.2 & 0.0049 \\
3 & 0.6 & 0.0098 \\
4 & 1.0 & 0.0196 \\
5 & 2.0 & 0.0392 \\
\hline
\end{tabular}

is divided into the cells of size of 0.025 . The near-wall layer: along the radius the size of the first cell is 0.0001 , the number of cells is 40 (at a gross factor of 1.10 normal to the wall). The total height of the near-wall layer is 0.01 . In azimuth, the profile is divided with a step of 0.002. The total number of MF-type grid cells is 191000 .

The second grid is more detailed and designated as M1 (Fig. 6, c-f). The near-wall layer thickness is 0.0015 (Fig. 6f). The near-wall step is $910^{-5}$ and increases with a gross factor of 1.1 normal to the wall. In the direction to the ring, the step is uniform, except the immediate area of the edge, and is equal to 640 cells on the profile. Four "circular" layers (Fig. 6.e) are assigned.

These layers consist of the cells of the uniform Cartesian grid, whose step increases when moving away from the profile (Table 3). The wake region is covered with the uniform Cartesian grid 4 in height and 15 in length. The grid step is 0.0196 (Fig. 6,d). In the longitudinal direction, the outer grid extends by 25 chords and in the vertical direction - by 40 chords. The computational domain is 5 chords away from the body, the outlet one -20 chords, and the upper and lower boundaries - 20 chords. The uniform grid step is 0.31 .

The M2- and M3-type grids are used for CFX and Star CCM+ computations.
The unstructured M2-type grid consists of triangular cells and has a layer of tetragonal cells near the body surface. The near-wall layer consists of 16 layers of cells increasing with a gross factor of 1.2. The size of the first layer is 0.0001. Edges are not smoothed. The "circular" layer of cells approaches the near-wall layer. The distance from the body to the external boundary of the ring is 0.15 . The cell size at this distance increases from 0.003 to 0.006 . The wake region is a rectangular area 4 in height and 21.5 in length. The mean size of the cell in it is 0.02 . The outer grid is 5 chords away from the inlet, 20 chords away from the outlet, and 20 chords away from the upper and lower boundaries. The mean size of grid fragments is 0.6 . The total number of grid cells is 560982 .

An M3-type unstructured grid consisting of polyhedral cells and having a prismatic layer near the body surface is realized in the StarCCM+ code. The near-wall layer consists of 16 layers of cells increasing with a gross factor of 1.2. The size of the first layer is 0.0002. Edges are not smoothed. The wake region is shaped as a trapezium 20 in height with an expansion angle of $15^{\circ}$. The minimal cell size in this region is 0.015 (these cells are located near the body) and the maximal size -0.028 . The outer grid is 5 chords away from the inlet, 20 chords from the outlet, and 20 chords from the upper and lower boundaries. The mean size of the boundary fragments of the grid is 0.3. The total number of grid cells is 325538.

\subsection{Validation. Comparison of the numerical predictions using multiblock $O$ - and H-type grids}

The VP2/3 code and the SST model corrected within the RLI approach are validated from the parametric studies of flow around the semi-circular profile by means of different-topology (O- and $\mathrm{H}-$ type) grids with a different discretization degree of the near-wall 


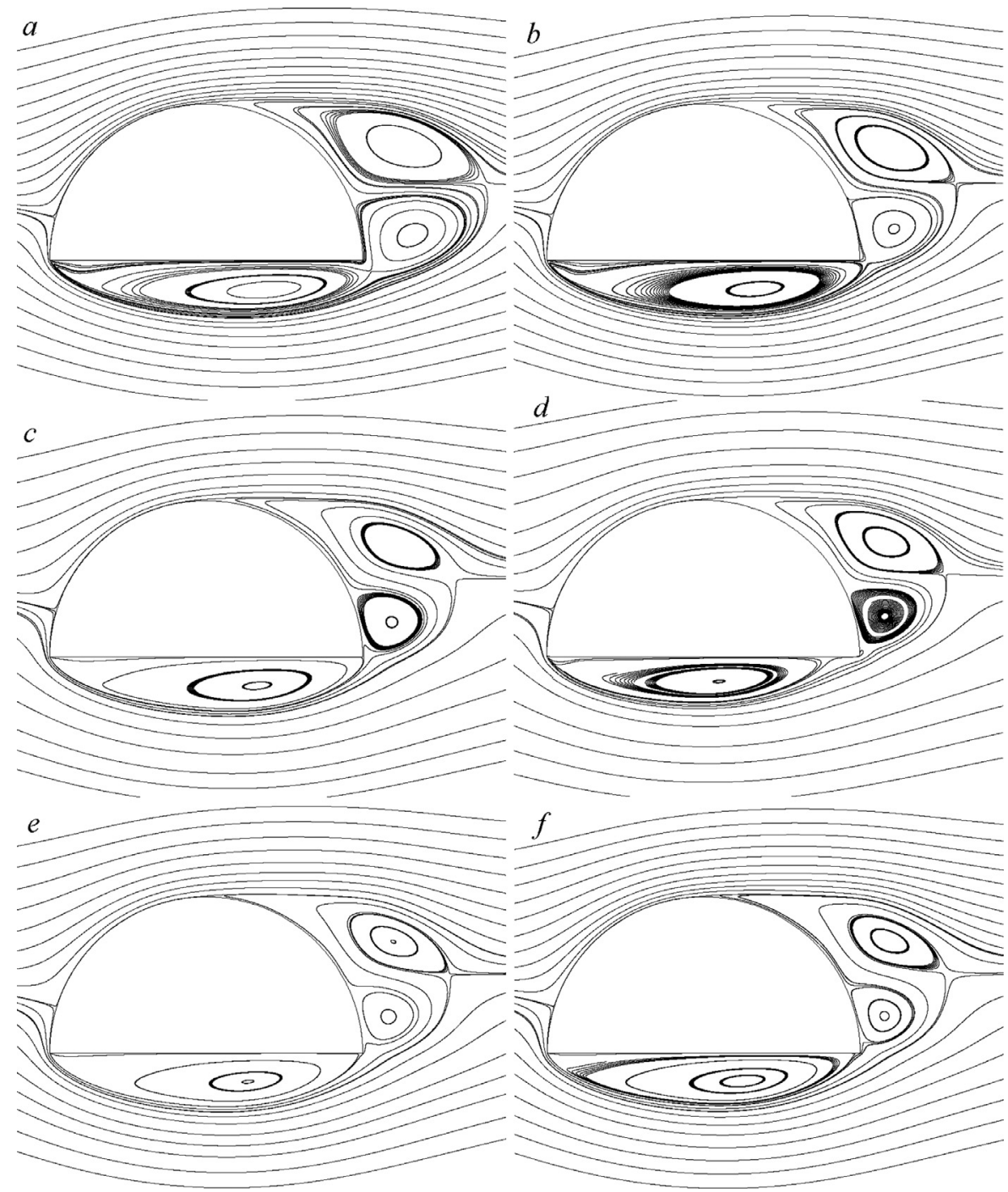

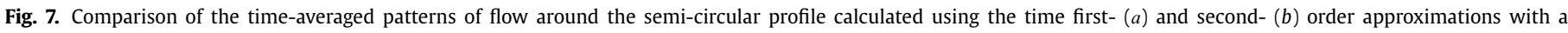

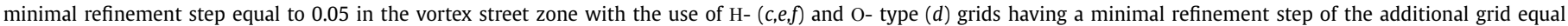
to 0.025 and different rounding radii: $R=0.002(a, b, c) ; 0.003(e) ; 0.005(d, f)$. VP2/3 code, SST model [19] corrected within the RLI approach.

\section{Table 4}

Comparison of the averaged loads and the extreme local characteristics on multiblock differenttopology and different-discretization grids, different integration schemes, and different rounding radii.

\begin{tabular}{lllllllll}
\hline Grid & $C_{x}$ & $C_{y}$ & $u_{\min }$ & $u_{\max }$ & $p_{\min }$ & $p_{\max }$ & $k_{\max }$ & $\mu_{\operatorname{tmax}}$ \\
\hline $\mathrm{O}$ & 0.569 & -0.87 & -0.590 & 1.559 & -1.319 & 0.505 & 0.113 & 0.0089 \\
$\mathrm{Ho}$ & 0.512 & -1.04 & -0.666 & 1.558 & -1.158 & 0.507 & 0.099 & 0.0138 \\
$\mathrm{H} 1(1 \mathrm{p})$ & 0.494 & -0.756 & -0.578 & 1.468 & -1.038 & 0.506 & 0.0961 & 0.0077 \\
$\mathrm{H} 1 \mathrm{a}(1 \mathrm{p})$ & 0.494 & -0.766 & -0.587 & 1.469 & -1.094 & 0.506 & 0.096 & 0.0084 \\
$\mathrm{H} 1 \mathrm{a}$ & 0.508 & -0.922 & -0.608 & 1.523 & -1.157 & 0.507 & 0.1003 & 0.0126 \\
$\mathrm{Hr}$ & 0.515 & -1.046 & -0.664 & 1.573 & 0.980 & 0.508 & 0.104 & 0.0133 \\
$\mathrm{H}^{*}$ & 0.512 & -1.063 & -0.672 & 1.573 & -0.985 & 0.508 & 0.1039 & 0.0139 \\
\hline
\end{tabular}

$1 \mathrm{p}$ - first-order time-integrated scheme.

area (the near-wall grid steps are $10^{-4}$ or $10^{-5}$ ) and of the wake region (the additional grid length is varied from 10 to 20 and the longitudinal grid step is refined from 0.005 to 0.025 ) using time different-order approximations (implicit first- and second-order approximation schemes of Euler (In Table 4, it is designated as 1p) and of Peyret - as SOUE). Moreover, the influence of the rounding radius of the profile edges is assessed on the grids refined in the vicinity of the edges. The comparisons are made using the $t^{*}$ dependences of integral loads $R_{x}$ and $R_{y}$ on the self-oscillatory period, the time-averaged distributions of $C_{p}$, fr and their oscillations $\left(p^{\prime}, f r^{\prime}\right)$, as well as the self-oscillatory period-averaged flow patterns, drag and lift coefficients, and extreme characteristics. Numerical $C_{p}$ predictions are compared with the measurements made in the present work.

Some computation data are summarized in Table 4 and are shown in Figs. 7 and 8. 


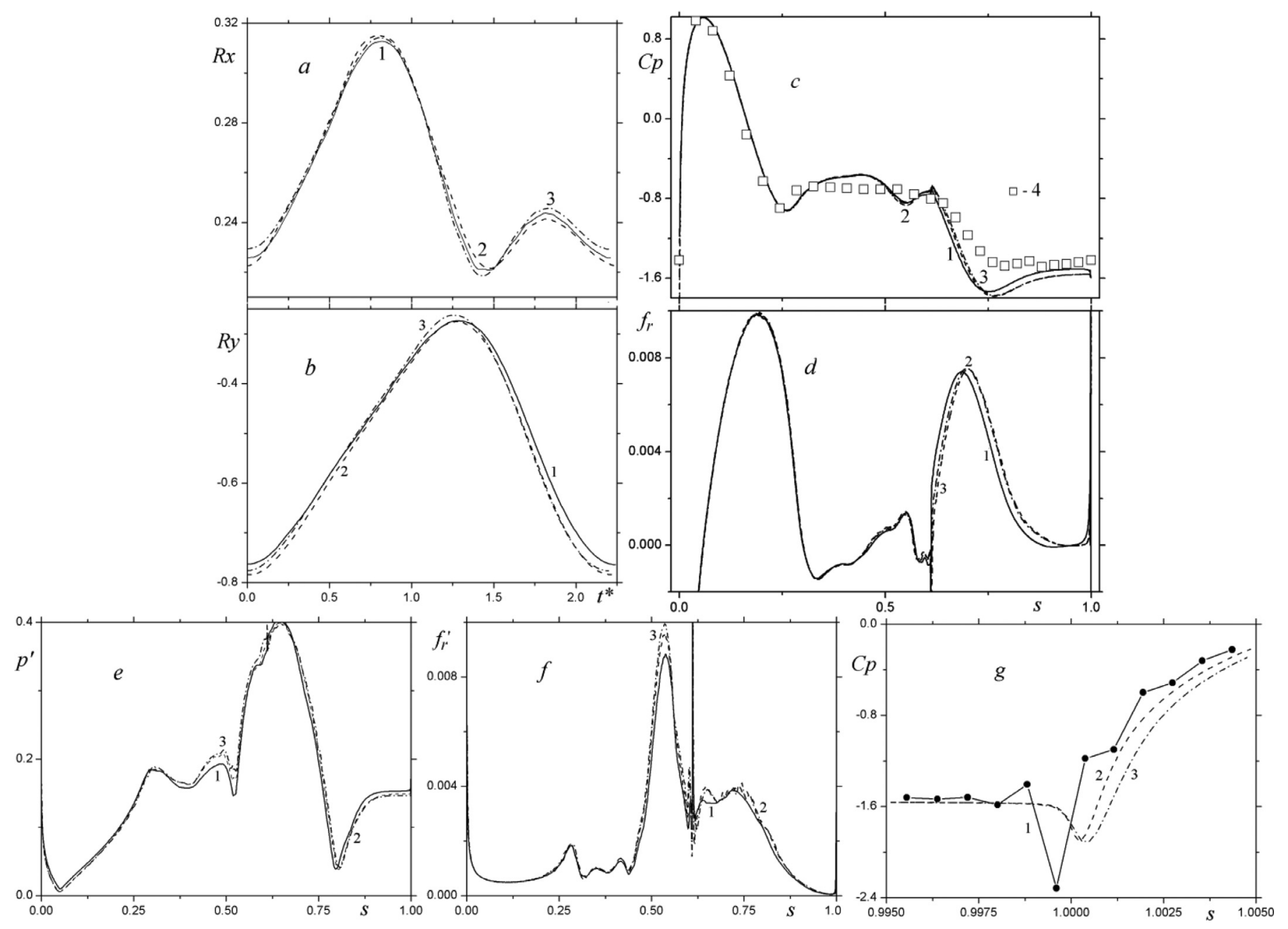

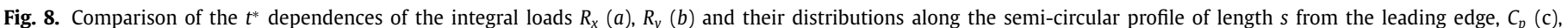

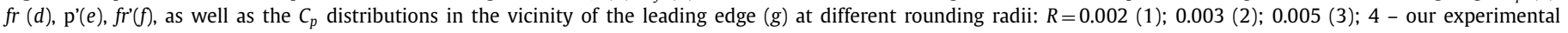
data.

Table 5

Designations are the same as in Figs. 9 and 10.

\begin{tabular}{lll}
\hline No. & Code & Grid \\
\hline 1 & SigmaFlow & M1 \\
2 & VP2/3 & M1 \\
3 & & $\mathrm{H}^{*} \mathrm{k}$ \\
4 & & $\mathrm{H}^{*}$ \\
5 & Fluent & $\mathrm{M} 1$ \\
6 & & $\mathrm{H}^{*} \mathrm{k}$ \\
7 & CFX & M2 \\
8 & StarCCM+ & M3 \\
9 & OpenFOAM & $\mathrm{M} 1$ \\
\hline
\end{tabular}

It should be noted that at $R=0.002$, the Ho-, H1-, and H1a-type grids in the vicinity of the leading edge are rather coarse (with two cells).

The number of cells of multiblock H-, H1- and H1a-type grids is twice smaller in comparison to Ho-, $\mathrm{Hr}^{-}$, and $\mathrm{H}^{*}$-type grids; the length of the additional refined grid (H1- and H1a-type grids) in the profile wake is twice shorter (10 instead of 20) than that of the remaining grids. In addition, the step of the additional refined grid (H1- and H1a-type grids) is 0.05 , whereas the step of additional multiblock Ho-, Hr-, and $\mathrm{H}^{*}$-type grids is 0.025 .

When analyzing the flow patterns, emphasis is made on the attachment of the flow separated from the leading edge (Fig. 7). A solution close to an exact one must be characterized by the flow attachment in the vicinity of the trailing edge.

From the results reported it follows that the second-order approximation significantly clarifies the solution of the problem, whereas the decrease in the near-wall grid step from $10^{-4}$ to $10^{-5}$ practically does not affect the solution.

The comparison of the results in Fig. 8 shows that varying the rounding radius does not significantly influence the distribution of integral and local flow parameters; however, to provide a monotonic pressure change (Fig. $8, \mathrm{~g}$ ) in the vicinity of sharp edges, the grid (H1r-, $\mathrm{H}^{*}$-type) should be condensed.

\subsection{Comparison of the numerical predictions using different} turbulence models and computational grids realized in different CFD codes

Figs. 9 and 10 illustrate the data for $R_{x}$ and $R_{y}$ on the selfoscillatory period obtained using both the SST model [18] (Fig. 9) and the SST model [19] (Fig. 10) with the consideration of the streamline curvature, as well as the computational grids realized in different codes.

It should be noted that the SST model [19] modified within the RLI approach is realized in the VP2/3 and SigmaFlow codes, the SST model [19] modified within the SM approach is implemented in the Fluent and CFX codes, whereas Durbin's correction is used in the StarCCM+ code. 

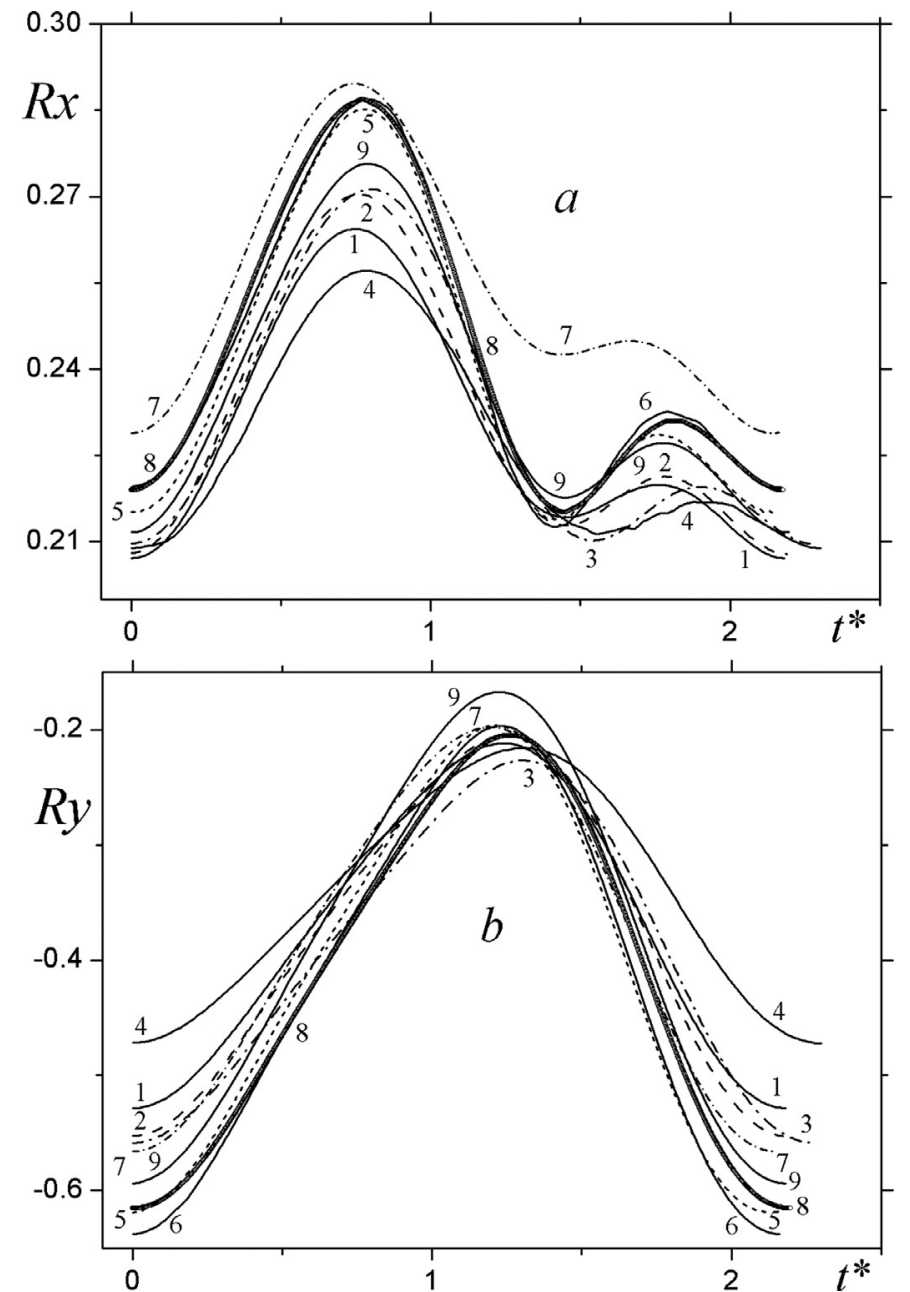

Fig. 9. Comparison of the $t^{*}$-dependent integral loads $R_{x}(a), R_{y}(b)$ using the standard SST model [19] as well as different computational grids realized in different codes (Table 5).

The time $t^{*}$ is taken from the time moments, at which $R_{x}$ and $R_{y}$ take local minimum values on the fluctuation period.

Figs. 11-13 summarize three groups of the predicted and experimental distributions of time-averaged surface pressure coefficients $C_{p}$ over the semi-circular profile. In the first group (Fig. 11), the numerical predictions are compared using the standard SST model [19] (VP2/3, SigmaFlow, and Fluent codes) and the SST model with the streamline curvature correction [19] modified within the RLI and SM approaches supplemented by the computation results obtained by the standard SST model [18] (VP2/3 code). The second group (Fig. 12) contains the numerical predictions with the use of the modified the SST model [19] (VP2/3, Sigma-Flow, Fluent, CFX, and StarCCM+codes) within the RLI and SM approaches and Durbin's correction.

The third group (Fig. 13) includes the predictions with the use of different turbulence models (SST, $\zeta-a, \zeta-f$ ) (VP2/3 and SigmaFlow codes). All graphs show the experimental data for quasitwo-dimensional flow around the semi-circular profile (with cutoff washers).

First, the inaccuracy of numerical predictions is explained by computational errors, when insufficiently detailed or overlapping grids are used, and second - by computational errors, when the chosen turbulence model is inadequate. The latter is especially associated with using the near-wall SST model [19] to analyze intense vortex flows, for which it was not calibrated. The experimen-
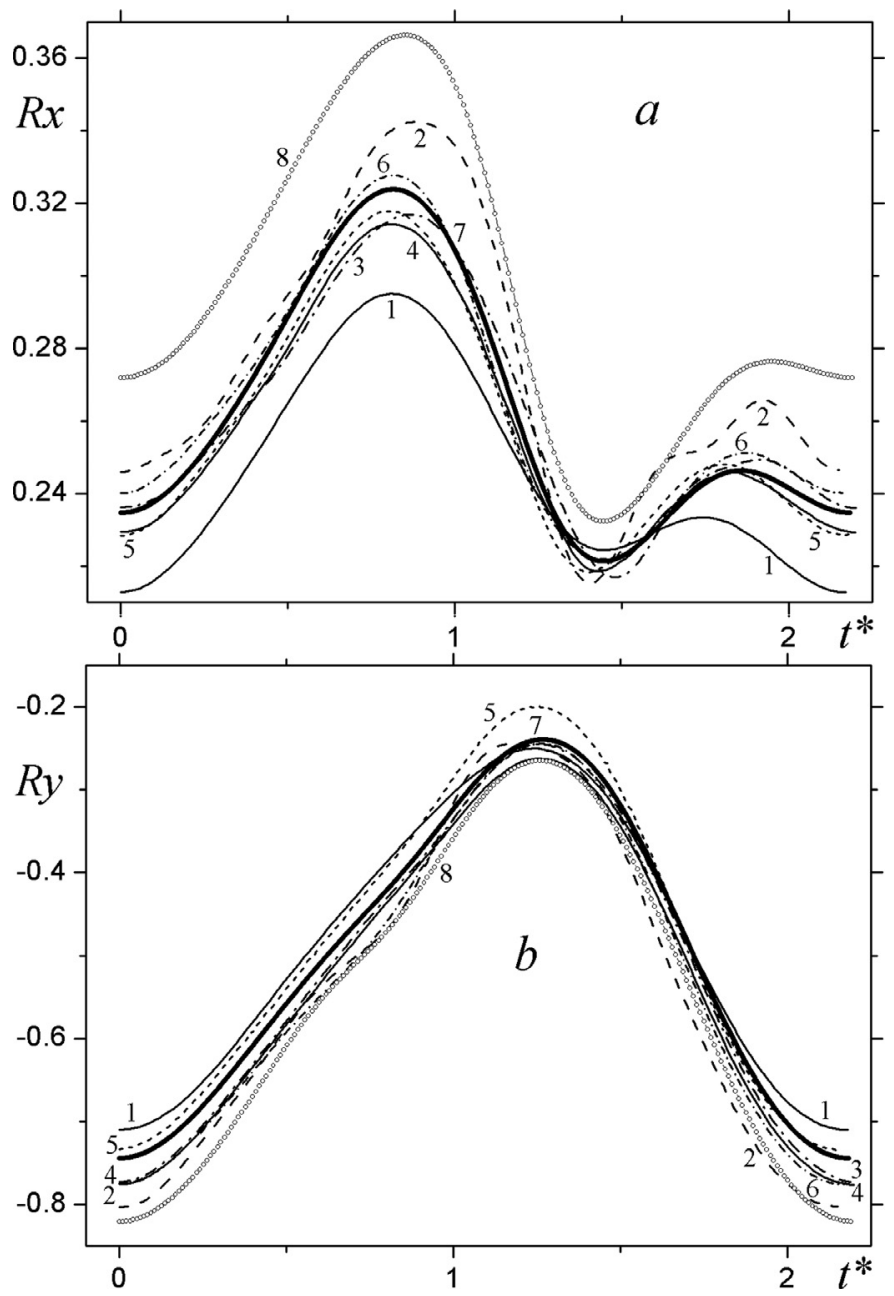

Fig. 10. Comparison of the $t^{*}$-dependent integral loads $R_{x}(a), R_{y}(b)$ using the modified SST model [19], as well as different computational grids realized in different codes (Table 5).

tal data for quasi-two-dimensional flow around the semi-circular profile (Figs. 11-13) serve as defined points to make comparison with the computation results. However, it is obvious that the influence of three-dimensional vortex structures, which intensify static pressure in the rarefaction zone in the vicinity of the lower part of the profile, remains substantial.

The behavior of integral and local loads on the semi-circular profile (Figs. 9-13) is markedly different for different types of grids, models, and codes. To assess the accuracy, it is useful to establish a topological similarity of the computation results and to note the density of the curves in certain, rather narrow bands of 0.02 in thickness for $R_{x}$ and of 0.1 for $R_{y}$. The analysis of the distributions $R_{x}\left(t^{*}\right)$ on the self-oscillatory period (Figs. 9 and 10,a) shows their two-mode character for the majority of numerical predictions, whereas the curves $R_{y}\left(t^{*}\right)$ in Figs. 9-10,b are single-mode in character.

A significant quantitative difference in the results obtained by means of the standard SST model [18] and the modified SST model $[19]$ is seen. Higher drag values and lower lift values are characteristic for the modified SST model [19], Overestimated predictions of time-averaged $C_{p}$ are observed at the lower wall of the profile (Fig. 11).

The standard SST model [19] using the multiblock overlapping grids (curves 4 in Fig. 9) allows the two-mode character of the de- 

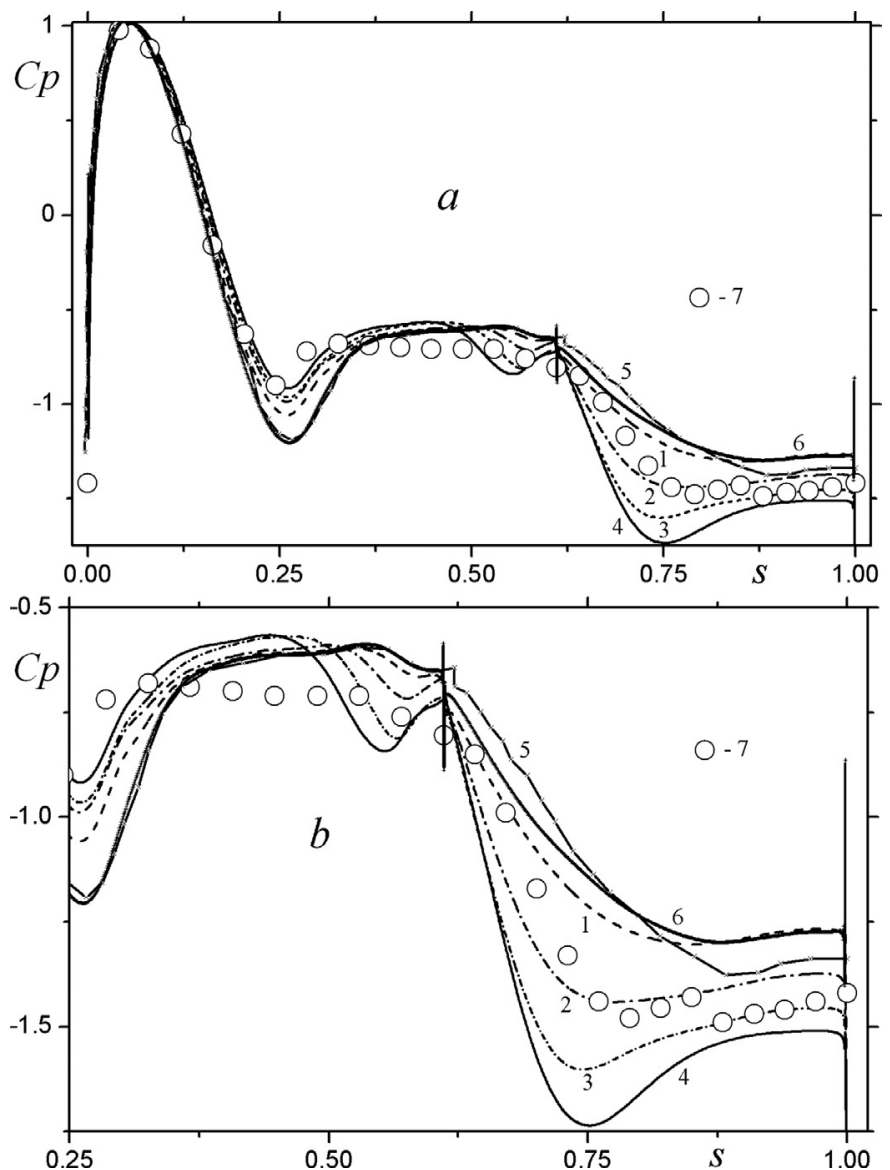

Fig. 11. Comparison of the time-averaged experimental and numerical distributions of the surface pressure coefficient over the semi-circular profile obtained using the standard model (VP2/3, SigmaFlow, Fluent) and the modified SST model considering the streamline curvature correction in normal $(a)$ and enlarged $(b)$ scale: 1 SST model [19] VP2/3 code; 2 - SST model [18] VP2/3 code; 3 - SST model modified within the SM approach, VP2/3 code; 4 - SST model modified within the RLI approach, VP2/3; 5 - SST model [19]. Fluent; 6 - SST model [19], SigmaFlow; 7 experiment (with cutoff washers shaped as discs, present work).

pendence $R_{x}\left(t^{*}\right)$ to be obtained; however, the quantitative differences from other dependences are big.

The $R_{x}\left(t^{*}\right)$ and $R_{y}\left(t^{*}\right)$ predictions using the structure-different composite M1- and $\mathrm{H}^{*}$-type grids in the VP2/3 and SigmaFlow codes appear to be very close.

The most high accuracy of the $R_{x}\left(t^{*}\right)$ and $R_{y}\left(t^{*}\right)$ results was found by the computations on very detailed grids in Fluent, StarCCM+, and OpenFOAM codes. A big difference from the mean dependences $R_{X}\left(t^{*}\right)$ is realized in the CFX code. If the dependences $R_{x}\left(t^{*}\right)$ obtained in SigmaFlow (curve 1), VP2/3 (curve 2), and StarCCM+ (curve 8) codes in Fig. 10,a are not taken into account, then for the modified SST model [19], the remaining dependences satisfactorily correlate with each other. The time-averaged $C_{p}$ distributions computed by the modified SST model with the streamline curvature correction in different codes appear to be very close.

The VP2/3 predictions on a $\mathrm{H}^{*}$-type multiblock grid, transformed into a composite $\mathrm{H}^{*} \mathrm{k}$-type one, appeared to be very close to the similar ones obtained in Fluent (composite M1- and $\mathrm{H}^{*} \mathrm{k}$ type grids) and in CFX (very detailed unstructured M2-type grid).

The numerical $R_{y}\left(t^{*}\right)$ predictions (Fig. 10, b) using the modified SST model and the considered different-type grids and codes are well consistent with each other. It is of significance to emphasize that unlike the SST model [19], MCT in combination with the SST model modified within the RLI approach has a high accuracy of
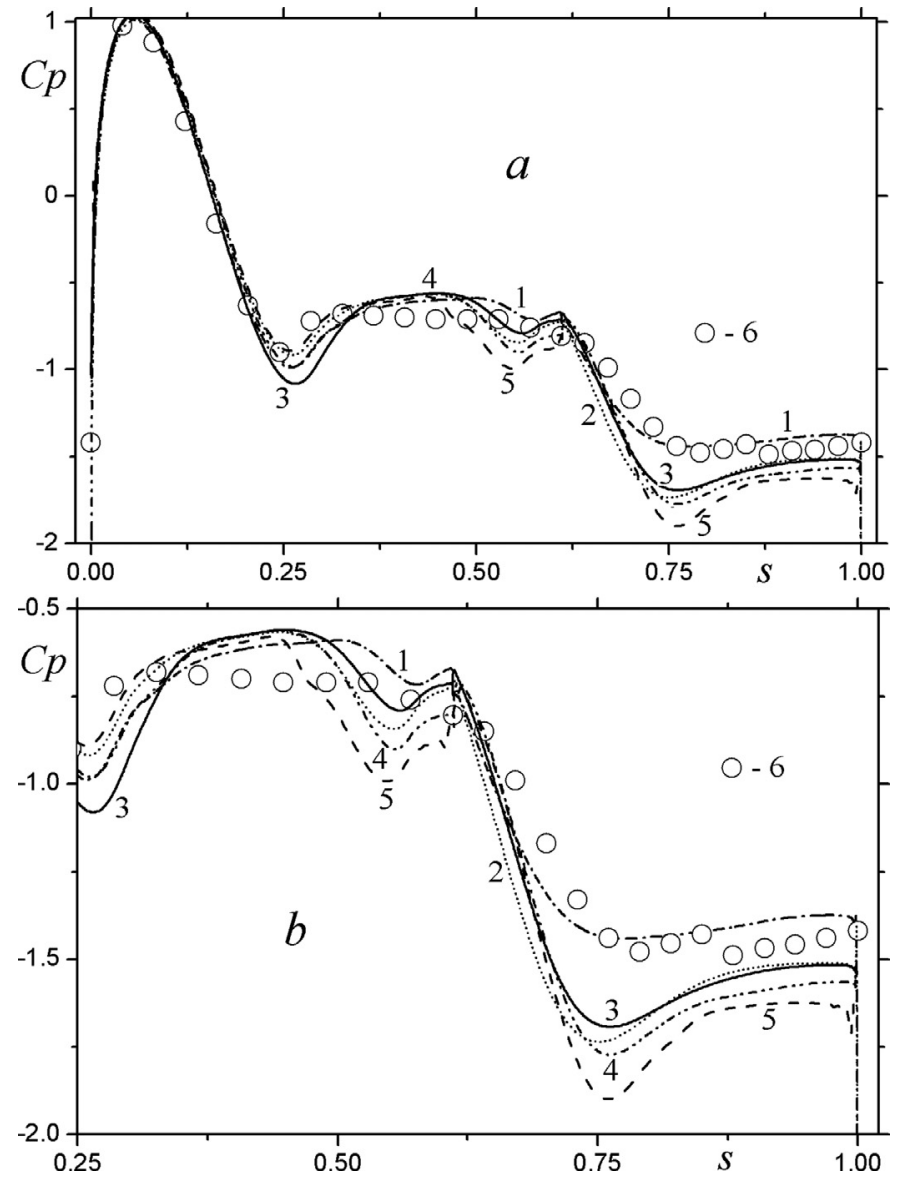

Fig. 12. Comparison of the time-averaged experimental and numerical distributions of the surface pressure coefficient over the semi-circular profile obtained using the modified SST models, VP2/3, SigmaFlow, Fluent, CFX, and StarCCM+codes in normal (a) and enlarged $(b)$ scale: 1 - SST model [18], VP2/3 code; 2 - SST model modified within the RLI approach, VP2/3; 3 - SST model modified within the RLI approach, SigmaFlow code; 4 - SST model modified within the SM approach, CFX code; 5 SST model modified with Durbin's correction, StarCCM+code; 6 - experiment (with cutoff washers shaped as discs, present work).

Table 6

Integral characteristics of averaged flow around the semi-circular profile on the self-oscillatory period computed by the standard SST model [19] with the use of various grids realized in different codes.

\begin{tabular}{lllll}
\hline Code & Grid & $C_{x}$ & $C_{y}$ & Sh \\
\hline VP2/3 & M1 & 0.464 & -0.756 & 0.457 \\
& $\mathrm{H}^{*} \mathrm{k}$ & 0.464 & -0.786 & 0.439 \\
& $\mathrm{H}^{*}$ & 0.454 & -0.686 & 0.435 \\
SigmaFlow & M1 & 0.460 & -0.738 & 0.459 \\
Fluent & M1 & 0.480 & -0.818 & 0.472 \\
& H*k & 0.484 & -0.840 & 0.463 \\
CFX & M2 & 0.508 & -0.758 & 0.466 \\
StarCCM+ & M3 & 0.446 & -0.690 & 0.459 \\
OpenFOAM & M1 & 0.486 & -0.826 & 0.455 \\
\hline
\end{tabular}

numerical predictions and practically does not differ from the predictions on composite grids.

The analysis of the time-averaged $C_{x}$ and $C_{y}$ computed by the standard SST model [19] (Table 6) shows that the mean $C_{x}$ and $C_{y}$ values estimated in all codes differ from the expected ones by approx. $10 \%$ for $C_{x}$ and by $25-30 \%$ for $C_{y}$. It is interesting to note that the lowest (in magnitude) predictions $\left(C_{x}=0.45 ; C_{y}=-0.69\right)$ have appeared to be the same using the multiblock overlapping $\mathrm{H}^{*}$-type grid realized in the VP2/3 code and the sufficiently fine unstructured M3-type grid realized in the StarCCM+ code. The use 

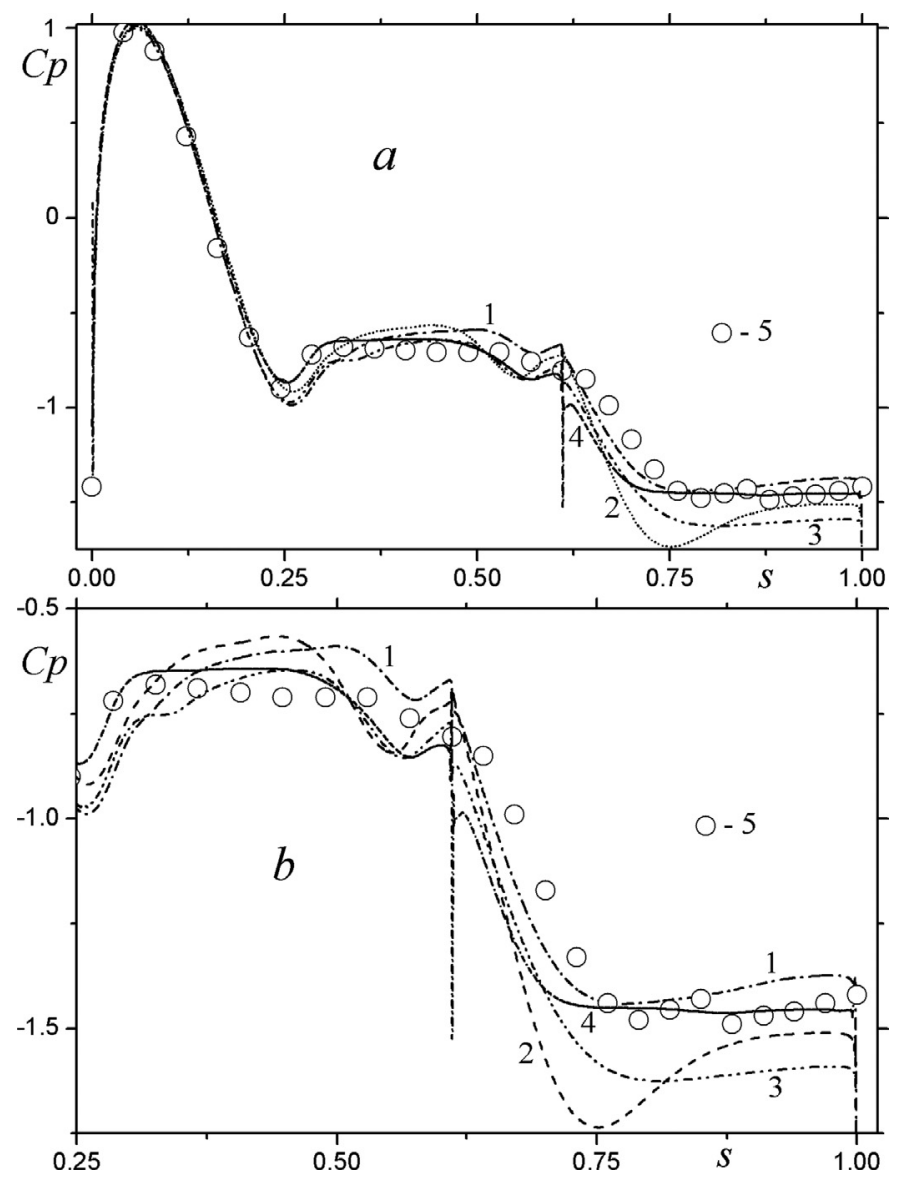

Fig. 13. Comparison of the time-averaged experimental and numerical distributions of the surface pressure coefficient over the semi-circular profile obtained using different SST, $\zeta-a$ and $\zeta-f$ turbulence models, VP2 $/ 3$ and SigmaFlow codes: $1-$ SST model [18], VP2/3 code; 2 - SST model modified within the RLI approach, VP2/3; $3-\zeta$-a model, SigmaFlow code; $4-\zeta-f$ model, SigmaFlow code; 5 - experiment (with cutoff washers shaped as discs, present work).

Table 7

Integral characteristics of averaged flow around the semi-circular profile on the selfoscillatory period computed by the modified SST model[19] on different codes and grids, as well as by the $\zeta-f$ and $\zeta-a$ models in the SigmaFlow code.

\begin{tabular}{|c|c|c|c|c|c|}
\hline Code & Correction for SST model [19] & Grid & $C_{x}$ & $C_{y}$ & Sh \\
\hline \multirow[t]{4}{*}{$\mathrm{VP} 2 / 3$} & RLI & MF & 0.514 & -0.880 & 0.467 \\
\hline & RLI & M1 & 0.550 & -1.072 & 0.465 \\
\hline & RLI & $\mathrm{H}^{*} \mathrm{k}$ & 0.522 & -1.032 & 0.453 \\
\hline & RLI & $\mathrm{H}^{*}$ & 0.514 & -1.048 & 0.455 \\
\hline \multirow[t]{2}{*}{ SigmaFlow } & RLI & MF & 0.488 & -0.886 & 0.469 \\
\hline & RLI & M1 & 0.492 & -0.962 & 0.461 \\
\hline \multirow[t]{3}{*}{ Fluent } & SM & MF & 0.514 & -0.920 & 0.472 \\
\hline & SM & M1 & 0.516 & -0.952 & 0.457 \\
\hline & SM & $\mathrm{H}^{*} \mathrm{k}$ & 0.530 & -1.048 & 0.463 \\
\hline CFX & SM & M2 & 0.526 & -0.995 & 0.458 \\
\hline \multirow[t]{2}{*}{ StarCCM+ } & Durbin & M3 & 0.528 & -1.100 & 0.457 \\
\hline & Four-parameter models & & & & \\
\hline \multirow[t]{4}{*}{ SigmaFlow } & $Z-a$ model & MF & 0.526 & -1.015 & 0.452 \\
\hline & & M1 & 0.541 & -1.092 & 0.440 \\
\hline & $Z-f$ model & MF & 0.541 & -0.962 & 0.413 \\
\hline & & M1 & 0.522 & -0.794 & 0.393 \\
\hline
\end{tabular}

of the Fluent and OpenFOAM codes has demonstrated the highest (in magnitude) close predictions. The CFX code has predicted a higher value of $C_{X}$.

Table 7 contains the testing computations by different modified SST models and various grids (multiblock overlapping, composite, and unstructured), as well as the predictions of the time-averaged aerodynamic coefficients of the streamlined semi-circular profile on the self-oscillatory period by the four-parameter $\zeta-f$ and $\zeta-a$ models.

The accuracy of numerical predictions, which approach the expected values of $C_{x}=0.52-0.54, C_{y}=-1.04-1.08$ and $\mathrm{Sh}=0.44-$ 0.46 (Table 1), increases with improving grid characteristics (when VP2/3, SigmaFlow, and Fluent codes are used).

It should be noted that the numerical predictions using the same grids (MF-type) realized in different codes (VP2/3, SigmaFlow, Fluent) are close.

When a multiblock overlapping $\mathrm{H}^{*}$-type grid and a composite $\mathrm{H}^{*} \mathrm{k}$-type grid based on it are used, the close results are obtained using the VP2/3 code. They well correlate with the numerical predictions with the implication of the $\zeta-a$ model.

The numerical results obtained on very detailed grids realized in the CFX and StarCCM+ codes most closely correspond to the experimental $C_{X}$ and $C_{y}$ data.

The solution accuracy of the two-dimensional test problem of flow around the semi-circular profile on the self-oscillatory period, obtained by the $\zeta-f$ model, gives way to similar predictions by the $\zeta-a$ model.

\section{Conclusion}

Experimental data on the pressure coefficient distribution over the semi-circular profile at zero angle of attack are obtained. The quasi-two-dimensional nature of flow around the profile is ensured by mounting the cutoff washers on the test model.

A new four-parameter $\zeta-a$ model is proposed and detailed. It is the analog of Hanjalić's known $\zeta-f$ model, on the basis of which the improved predictions are obtained in the present work. The features of the algorithms and the computational methods realized in the SigmaFlow, VP2/3, Fluent, CFX, StarCCM+ and OpenFOAM codes are analyzed. Special attention is paid to comparing grid structures; along with regular unstructured grids, several-type multiblock different-scale structured overlapping grids of simple topology are considered. Based on them composite analogs are built, in which the overlapping zones of grids are replaced by unstructured fragments; the share of cells of structured fragments prevails. Special attention is paid to multiblock overlapping grids that are a basis of the multiblock computational technology realized in the VP2/3 code.

A detailed comparison is made of the testing computations and experimental results of incompressible fluid flow around the semicircular profile at the zero angle of attack and $\mathrm{Re}=50000$ for different scheme parameters (grids of different details and topology - H- and O-type grids, discretization schemes of the convective terms of the equations, the accuracy order of time integration schemes, initial conditions, rounding radius), the standard SST turbulence models [18] and [19], the SST model [19] with the streamline curvature correction modified within the Rodi-Leschziner-Isaev approach and the Smirnov-Menter approach; the four-parameter $\zeta-a$ and $\zeta-f$ models; six codes (SigmaFlow, VP2/3, Fluent, CFX, StarCCM+ and OpenFOAM). It is shown that the numerical predictions satisfactory in accuracy are obtained on grids with the cell number equal to 300-500 thousands, with a near-wall step of $10^{-4}$ of the chord, as well as using the second-order approximation scheme at a time step of $10^{-2}$, with the grid refinement in the near wake at a grid step of 0.025 and the length of a selected wake region of no less than 20 chords.

The standard near-wall SST models implemented in all considered codes predict substantially underestimated (up to 30\%) loads at the semi-circular profile, although the standard SST model [18], unlike the SST model [19], yields slightly better results since it reproduces separated and vortex flows with less error. Streamline curvature corrections substantially decrease the level of eddy viscosity in vortex cores, vortex flow enhancement, and favor a better 
agreement between numerical predictions and experimental data. The RLI approach, where the semi-empirical constant $C_{c}=0.02$ inversely depends on the turbulent Richardson number in the correction formula for definition of eddy viscosity, appears to be preferable to the SM approach. A better agreement of the numerical predictions by means of the SST model [19] modified within the RLI approach, which have been obtained by different codes (VP2/3 and SigmaFlow) and grids (multiblock and composite), is also shown.

The comparison of the SigmaFlow, VP2/3, Fluent, CFX, StarCCM+, and OpenFOAM codes has revealed a large scatter both of the numerical predictions of $R_{x}\left(t^{*}\right)$ and $R_{y}\left(t^{*}\right)$, where the time $t^{*}$ is taken from the start of the self-oscillatory period at minimum load, and of time-averaged $C_{x}$ and $C_{y}$ using the near-wall SST model [19]. This is done with the use of the standard near-wall SST model [19] which is one of the basic turbulence models. At the same time, the use of the modified SST models with the correction for streamline curvature strongly improved the prediction of characteristics, in particular the $R_{y}\left(t^{*}\right)$ predictions became much closer. It appeared that the use of the SST model modified within the RPL approach and MCT realized in the VP2/3 code yields the numerical predictions that slightly differ from those obtained on composite grids.

The performed comparison of the semi-empirical models of different type (two-parameter SST model modified within the RLI approach, four-parameter $\zeta-a$ and $\zeta-f$ turbulence models) showed a satisfactory agreement of the results obtained on different grids in different codes (VP2/3 and SigmaFlow). The preferable results were obtained using both the SST model [19] modified within the RLI approach and the proposed $\zeta-a$ model.

\section{Acknowledgment}

The work has been supported by the Government of the Russian Federation according to Grant no. 14.Z50.31.0003 (testing and physical experiments); Leading scientist - Prof. S. Isaev, Kazan Scientific Research Technical University - KAI as well as of the Russian Foundation for Basic Reseach under the Project No. 18-01-00210.

\section{References}

[1] Takizawa N Okada $\mathrm{N}$ Iwasaki $\mathrm{F}$ Wind-tunnel investigation of the pressure distribution and deduced characteristics of semi-circular cylinder in the vicinity of critical Reynolds number. Tech Rep Nation Aerospace Lab (Tokyo) 1985;871:1-43

[2] Yamagata T. Saito N Fujisawa N. Aeolian tone from a semi-circular cylinder in a stream. Flow Control Meas Visual 2016:4:30-7.

[3] Isaev SA Miau J-j, Sudakov AG, Usachov AE. Analysis of extremal lift behavior of a semi-circular airfoil in a turbulent airfoil at a near-zero angle of attack. Techn Phys Letters 2015;41:737-9.

[4] A. Savitsky, L. Schukin, V. Karelin et al., Method for control of the boundary layer on the aerodynamic surface of an aircraft, and the aircraft provided with the boundary laver control system. United States Patent No. 5417391. 1995.

[5] Baranov PA Isaev SA Prigorodov YuS, Sudakov AG Controlling the turbulent flow past a thick airfoil by means of flow enhancement in vortex cells using suction from central body surfaces. Fluid Dyn 2003:38:387-96.

[6] Isaev SA Baranov PA Sudakov AG Ermakov AM Modeling the increase in aerodynamic efficiency for a thick (37.5\% chord) airfoil with slot suction in vortex cells with allowance for the compressibility effect. Tech Phys Lett 2015:41:76-9.

[7] Isaev SA Baranov PA, Kudrjavtsev NA Zhukova YuV. Numerical simulation of unsteady heat exchange at a turbulent flow around a circular cylinder: part 1, methodic studv. Thermophys Aeromech 2005:12:27-38.

[8] Isaev SA Baranov PA, Kudrjavtsev NA Zhukova YuV. Numerical simulation of unsteady heat exchange at a turbulent flow around a circular cylinder: part 2, autooscillatory regime analvsis. Thermophvs Aeromech 2005:12:253-65.

[9] Isaev SA Baranov PA, Kudryavtsev NA Lysenko DA Usachov AE. Comparative analysis of the calculation data on an unsteady flow around a circular cylinder obtained using the VP2/3 and Fluent packages and the Spalart-Allmaras and Menter turbulence models. I Eng Phys Thermophys 2005;78:1199-213.

[10] Baranov PA Guvernyuk SV, Isaev SA Soudakov AG Usachov AE. Simulation of periodical structures in the airfoil wake. TsAGI Sci I 2014:45:273-92.

[11] Ferziger JH Perıc M. Computational methods for fluıd dynamıcs. Berlın, Heldelberg; 1999.
[12] Kao K-H, Liou M-S. Advance in overset grid schemes: from Chimera to DRAGON grids. AIAA I 1995:33:1809-15.

[13] Zheng Y Liou M-S A novel approach of three-dimensional hybrid orid methodology: part 1, grid generation. Comp Methods Appl Mech Eng 2003:192:4147-71

[14] Isaev SA, Zhdanov VL Niemann H-J Numerical study of the bleeding effect on the aerodynamic characteristics of a circular cylinder. J Wind Eng Ind Aerodyn 2002:90:1217-26.

[15] Isaev SA Baranov PA Usachov AE Multiblock computational technologies in the VP2 $/ 3$ package on aerothermodynamics. Saarbrucken: LAP LAMBERT Academic Publishing: 2013

[16] Isaev SA Sudakov AG, Baranov PA. Zhukova YuV, Usachov AE Analysis of errors of multiblock computational technologies by the example of calculating a circulation flow in a square cavity with a moving cover at $R e=1000$. J Eng Phys Thermophys 2013:86:1134-50.

[17] Bobyshev VK Isaev SA. Numerical study of the effects of the current turbulence on the flow along cylinder with a front disk situated. J Eng Phys Thermophys 1990:58:556-72

[18] Menter FR Zonal two equation $k-\omega$ turbulence models for aerodynamic flows. AIAA Paper 1993:93-2906 №.

[19] Menter FR Kuntz M Langtry R Ten years of industrial experience with the SST turbulence model. Turbulence, heat and mass transfer 4. Hanjalic K, Nogano Y, Tummers M. editors. Begell House Inc.: 2003.

[20] Isaev SA Baranov PA Zhukova YuV Usachov AE Kharchenko VB Correction of the shear-stress-transfer model with account for the curvature of streamlines in calculating separated flows of an incompressible viscous fluid. J Eng Phys Thermophys 2014:87:1002-15.

[21] Isaev SA. Baranov PA Usachov AE, Zhukova YuV Vysotskaya AA Malyshkin DA Simulation of the turbulent air flow over a circular cavity with a variable opening angle in an U-shaped channel. J Eng Phys Thermophys 2015:88:902-17.

[22] Isaev SA Baranov PA, Zhukova YuV, Kalinin EI Miau II. Verification of the shear-stress transfer model and its modifications in the calculation of a turbulent flow around a semicircular airfoil with a zero angle of attack. J Eng Phvs Thermophvs 2016:89:73-89.

[23] Smirnov PE. Menter F Sensitization of the SST turbulence model to rotation and curvature by applying the Spalart-Shur correction term. In: Proc. ASME Turbo Expo Conf.: 2008. No. GT2008-50480.

[24] Spalart PR Shur ML. On the sensitization of turbulence models to rotation and curvature. Aerospace Sci Technol 1997:1:297-302.

[25] Durbin PA. On the $k-3$ stagnation point anomaly. Int J Heat Fluid Flow 1996;17:89-90.

[26] Hanjalíc K, Popovac M Hadżıabdić M. A robust near-wall elliptıc relaxation eddy viscosity turbulence model for CFD. Int J Heat Fluid Flow 2004:25:1047-51.

[27] Manceu R. Hanjalic K Elliptic blending model: a new near-wall Reynolds-stress turbulence closure. Phys Fluids 2002:14:744-54.

[28] Manceu R. Recent progress in the development of the elliptic blending Revnolds-stress model. Int I Heat Fluid Flow 2015:51:195-220.

[29] Speziale CG. Sarkar S Gatski TB. Modeling the pressure-strain correlation of turbulence: an invariant dynamical system approach. J Fluid Mech 1991:227:245-72.

[30] Launder BE. Spalding DB. The numerical computation of turbulent flow. Comp Meth Appl Mech Eng 1974;3:269-89.

[31] Leschziner M, Rodı W. Calculation of annular and twin parallel jets using varıous discretization schemes and turbulence-model variations. Trans ASME J Fluids Eng 1981:103:352-65.

[32] Menter F. Ferreira JC. Esch T, Konno B. Turbulence model with improved wall treatment for heat transfer predictions in gas turbines. In: Proc. Int. Gas Turbine Congress 2003, Tokyo; 2003.

[33] Durbin PA Near-wall turbulence closure modeling without "damping functions". Theor Comp Fluid Dyna 1991:3:1-13.

[34] Billard F Laurence D A robust $k-e-u 2 / k$ elliptic blending turbulence model applied to near-wall, separated and buoyant flows. Int I Heat Fluid Flow 2012;33:45-58.

[35] ANSYS Fluent Theory Guide. Release 15.0. Southpointe: ANSYS, Inc.; 2013

[36] ANSYS CFX-Solver, Release 15.0: Theory Guide, Southpointe, 2013.

[37] STAR-CCM+ Documentation, Version 10.02 @ 2015 CD-adapco ${ }^{\mathrm{TM}}$.

[38] OpenFOAM, User Guide version 3.0.1, December 2015.

[39] http://www.itp.nsc.ru/applied-exploit/programmniv-paket-sigmaflow.

[40] Van Doormaal JP. Raithby GD Enhancement of the SIMPLE method for predicting incompressible fluid flow. Num Heat Transf 1984:7:147-63.

[41] Jasak Hi Error analysis and estimation for the finite volume method with applications to fluid flows, Thesis submitted for the Degree of Doctor of Philosophy of the University of London and Diploma of Imperial College of Science. Tech Med 1996

[42] Isaev SA Kudryavtsev NA Sudakov AG. Numerical modeling of a turbulent incompressible viscous flow along bodies of a curvilinear shape in the presence of a mobile shield. I Eng Phys Thermophys 1998:71:613-26.

[43] Rhie CM. Chow WL A numerical study of the turbulent flow past an isolated airfoil with trailing edge separation. AIAA I 1983:21:1525-32.

[44] Pascau A. Garcia N. Consistency of SIMPLEC scheme in collocated grids. V European Conference on Computational Fluid Dynamics ECCOMAS CFD 2010 2010.

[45] Leonard BP. A stable and accurate convective modeling procedure based on quadratic upstream interpolation. Comp Meth Appl Mech Eng 1979;19:59-98. 
[46] Van Leer B. Towards the ultimate conservative difference scheme V. A second order sequel to Godunov's method. I Comp Phys 1979:32:101-36.

[47] Saad Y. Iterative methods tor sparse linear systems. 2nd ed. Philadelphia: Soc. Ind. App. Math; 2003.

[48] Peyret R, Taylor TD. Computational methods for fluid flow. Springer-Verlag, New York Inc.; 1983.

[49] D. Demidov, AMGCL: $C++$ library for solving large sparse linear systems with algebraic multigrid method, http://amgcl.readthedocs.org/.
[50] Raw MJ Robustness of coupled algebraic multigrid for the Navier-Stokes equations. AIAA Paper 1996 No. 96-0297.

[51] Barth T] Jesperson DC The design and application of upwind schemes on unstructured meshes. AIAA Paper 1989 No. 89-0366.

[52] Weiss JM Maruszewski JP. Smith WA. Implicit solution of preconditioned Navier-Stokes equations using algebraic multigrid. AIAA I 1999:37:29-36.

[53] Roe PL Characteristic-based schemes for the Euler equations. Annu Rev Fluid Mech 1986;18:337-65. 